\title{
Evolutionary origins, molecular cloning and expression of carotenoid hydroxylases in eukaryotic photosynthetic algae
}

\author{
Hongli Cui ${ }^{1,2 \dagger}$, Xiaona Yu ${ }^{3 \dagger}$, Yan Wang ${ }^{1}$, Yulin Cui ${ }^{1}$, Xueqin $\mathrm{Li}^{4}$, Zhaopu Liu ${ }^{3}$ and Song Qin ${ }^{1 *}$
}

\begin{abstract}
Background: Xanthophylls, oxygenated derivatives of carotenes, play critical roles in photosynthetic apparatus of cyanobacteria, algae, and higher plants. Although the xanthophylls biosynthetic pathway of algae is largely unknown, it is of particular interest because they have a very complicated evolutionary history. Carotenoid hydroxylase (CHY) is an important protein that plays essential roles in xanthophylls biosynthesis. With the availability of 18 sequenced algal genomes, we performed a comprehensive comparative analysis of chy genes and explored their distribution, structure, evolution, origins, and expression.

Results: Overall 60 putative chy genes were identified and classified into two major subfamilies (bch and cyp97) according to their domain structures. Genes in the bch subfamily were found in 10 green algae and 1 red alga, but absent in other algae. In the phylogenetic tree, bch genes of green algae and higher plants share a common ancestor and are of non-cyanobacterial origin, whereas that of red algae is of cyanobacteria. The homologs of cyp97a/c genes were widespread only in green algae, while cyp97b paralogs were seen in most of algae.

Phylogenetic analysis on cyp97 genes supported the hypothesis that cyp97b is an ancient gene originated before the formation of extant algal groups. The cyp97a gene is more closely related to cyp97c in evolution than to cyp97b. The two cyp97 genes were isolated from the green alga Haematococcus pluvialis, and transcriptional expression profiles of chy genes were observed under high light stress of different wavelength.

Conclusions: Green algae received a $\beta$-xanthophylls biosynthetic pathway from host organisms. Although red algae inherited the pathway from cyanobacteria during primary endosymbiosis, it remains unclear in Chromalveolates. The $a$-xanthophylls biosynthetic pathway is a common feature in green algae and higher plants. The origination of cyp97a/c is most likely due to gene duplication before divergence of green algae and higher plants. Protein domain structures and expression analyses in green alga H. pluvialis indicate that various chy genes are in different manners response to light. The knowledge of evolution of chy genes in photosynthetic eukaryotes provided information of gene cloning and functional investigation of chy genes in algae in the future.
\end{abstract}

Keywords: Carotenoid hydroxylase, Xanthophylls biosynthesis, Structure and evolution, Molecular cloning, Expression profiles, Algae

\footnotetext{
* Correspondence: sqin@yic.ac.cn

${ }^{\dagger}$ Equal contributors

${ }^{1}$ Key Laboratory of Coastal Biology and Biological Resources Utilization, Yantai Institute of Coastal Zone Research, Chinese Academy of Sciences, Yantai 264003, People's Republic of China

Full list of author information is available at the end of the article
} 


\section{Background}

Carotenoids are isoprenoids which derived from the precursor molecule isopentenyl pyrophosphate and its isomer dimethylallyl diphosphate. All photosynthetic organisms including cyanobacteria, algae, and higher plants synthesize carotenoids [1,2]. Carotenoids are divided into two major groups: carotenes, which are enriched in the centers of photosystem reaction [3,4], and xanthophylls (including lutein, zeaxanthin, antheraxanthin, violaxanthin, and neoxanthin), oxygenated carotenoids that serve various functions in photosynthetic organisms and are essential for survival of the organism [5-8]. Of xanthophylls, lutein is the most abundant xanthophyll in all plant photosynthetic tissues, where it plays an important role in assembly and function of light harvesting complexes II (LHC II) [6-8]. Zeaxanthin is a structural isomer of lutein and a mainly component of the non-photochemical quenching (NPQ) mechanism involved in protecting the organism from photodamage [9-11].

Untangling the phylogenomics of xanthophylls biosynthesis in eukaryotic algae requires basic knowledge of their evolutionary histories. Three algal phyla, the Rhodophyta (red algae), Glaucophyta, and Viridiplantae (land plants and green algae) acquired their plastids during primary endosymbiosis starting a heterotrophic eukaryote as a host and a phototrophic cyanobacterium as an endosymbiont [12-18]. Viridiplantae is comprised of two major evolutionary lineages that split in early: the Chlorophyta (Chlorophyceae, Ulvophyceae, Trebouxiophyceae, and Prasinophyceae) and the Streptophyta (Charophyte and Embryophytes) [19-21]. Subsequently, secondary endosymbiosis from green or red algae occurred, producing the extant diverse algal groups (for reviews, see $[22,23])$. Algae comprise a paraphyletic and polyphyletic group [24], as they do not include all the descendants of the last universal ancestor nor do they all descend from a common algal ancestor, although their plastids seem to have a single origin [25]. From an evolutionary perspective, carotenoid biosynthesis in photosynthetic organisms has become increasingly more complex and diverse [26]. Some photosynthetic bacteria, e.g., cyanobacteria contain carotenoids with $\beta$-rings (e.g. $\beta$-carotene), and many of them produce various mono- or di-hydroxy xanthophyll derivatives (e.g. zeaxanthin) $[27,28]$. In addition, pathway bifurcation occurred in some red algae and cyanobacteria (i.e. Acaryochloris and Prochlorococcus), and all green algae, and higher plants at the level of lycopene cyclization, yielding $\alpha$-carotene from which $\alpha$-carotenederived xanthophylls (e.g. lutein) are synthesized [29-32]. As a consequence, the origins and evolution of xanthophylls biosynthetic pathway in algae lineages is of particular interesting.
Carotenoid hydroxylases (CHYs) comprise an important hydroxylase protein family performing the key enzymatic steps, hydroxylation reactions for $\alpha$ - and $\beta$-branch xanthophylls biosynthesis in photosynthetic organisms [33]. In higher plants, production of $\alpha$-xanthophylls from $\alpha$-carotene requires one $\beta$ - and one $\varepsilon$-ring hydroxylation, while synthesis of $\beta$-xanthophylls from $\beta$ carotene requires two $\beta$-ring hydroxylations. Two classes of structurally unrelated enzymes catalyze these ring hydroxylations: two heme-containing cytochrome P450 hydroxylases (CYP97A3 and CYP97C1) and a pair of nonheme/di-iron hydroxylases ( $\mathrm{BCH} 1$ and $\mathrm{BCH} 2)$ [34-37]. Two $\beta$-ring hydroxylations in $\beta$-carotene are mediated by either P450-type CYP97A or BCH enzyme, during which zeaxanthin is produced [34-36]. Hydroxylations of $\beta$-ring and $\varepsilon$-ring in $\alpha$-carotene are performed by CYP97A and CYP97C, respectively, producing lutein [37]. It is unknown why two different $\beta$-ring hydroxylases could have been maintained throughout the evolution. Probably, their own respective activities are not entirely interchangeable [38]. Different chy genes in higher plants are up-regulated often differentially, depending on environmental conditions or developmental stage of various tissues $[6,8,26,33,36-40]$. For algae, however, the research on xanthophylls biosynthesis is still in its infancy. For unicellular green algae, $b c h$ genes were investigated such as in Haematococcus pluvialis and Chlamydomonas reinhardtii [41-43]. Although lutein and its derivatives are detected in Rhodophyta (macrophytic type), Cryptophyta, Euglenophyta, Chlorarachniophyta, and Chlorophyta, enzymes involving in hydroxylation of $\alpha$-carotene remains unknown [44]. Recently, a new P450 protein responsible for the hydroxylation of $\varepsilon$-ring of $\alpha$-carotene in Arabidopsis thaliana was reported [37,45]. In addition, two genes encoding cytochrome P450-type carotenoid hydroxylases (cyp97a4 and cyp97c2) were isolated from Oryza sativa and their functions were investigated in a $\beta$-carotene producing Escherichia coli strain [35]. Moreover, 25 cytochrome $\mathrm{P} 450$ oxidoreductases have been discovered so far in the green alga Ulva linza [46]. Therefore, it is evident that P450-type monooxygenases, in addition to non-heme hydroxylases such as $\mathrm{BCH}$, are involved in carotenoid hydroxylation, which identified one of the missing pieces of carotenoid biosynthetic enzyme and provided valuable clues to study chy genes in algae in the future.

Recently, the genome sequences of a number of microalgae became available from the DOE Joint Genome Institute (http://genome.jgi.doe.gov/). Proteins coding sequences of each filtered model of these algae genomes with representatives from very different groups such as green algae, red algae, diatoms, and Haptophytes was performed to identify their chy genes. Details of the completeness of genome sequences used 
in this study were taken from DOE Joint Genome Institute project list (project list: http://genome.jgi.doe.gov/ genome-projects/) [see Additional file 1: Table S1]. A BLASTp-HMMER-plus-phylogeny reconstruction approach was employed to analyze CHYs in focus of their distribution, structure, evolution, origins, and expression. In order to study the evolutionary histories of xanthophylls biosynthetic pathway from cyanobacteria to modern algae, candidate genes were then compared with known sequences of other organisms. Based on the predicted putative $\mathrm{CHY}$-encoding genes, two full-length sequences of CYP97-encoding cDNA (Haecyp97a and Haecyp97b) were isolated from high-astaxanthin-production green alga $H$. pluvialis strain Flotow 1844 [42]. Expression profiles of different chy genes under high light stress of different wavelength were observed by means of relative quantitative real-time RT-PCR (Reverse Transcription-Polymerase Chain Reaction). Light is considered an effective stimulus inducing the expression of carotenoid biosynthesisrelated genes and the astaxanthin accumulation in economic green alga $H$. pluvialis $[47,48]$. These studies provide significant insight into the origins and evolution of chy genes in photosynthetic eukaryotes and provide information for further gene cloning, functional characterization, and expression analysis of chy genes in algae. A better understanding of algal CHYs can help us to comprehend the roles of CHYs in xanthophylls biosynthetic under different adverse conditions.

\section{Results and discussion}

\section{Identification, classification, and distribution of $\mathrm{CHY}$ proteins}

18 algal nuclear genomes were examined for putative genes of $\mathrm{CHY}$ proteins. A summary of algal genes putatively encoding CHYs is shown in Table 1 , and the classification and distribution of candidate genes, and hypothesized xanthophylls biosynthetic pathways across organisms are given in Figure 1. A total of 11 and 49 putative genes encoding $\mathrm{BCH}$ and $\mathrm{CYP} 97$, respectively, were predicted and annotated from 18 complete or incomplete eukaryotic algal genomes. Deduced protein sequences of genes encoding CHYs from the 18 algal genomes are shown in the Additional file [see Additional file 2]. Among the 18 eukaryotic photosynthetic algae, red algae possessed a $b c h$ homolog (CrtR-type) only. Hydroxylation of $\beta$-carotene in different organisms is primarily carried out by three gene subfamilies of the non-heme/di-iron monooxygenase superfamily: $b c h$ genes of higher plants and green algae; $b c h$ genes of nonphotosynthetic bacteria; and $C r t R$ genes of cyanobacteria [37]. Green algae inhabiting freshwater (Chlorophyceae: C. reinhardtii and $V$. carteri; Trebouxiophyceae: $C$. sp. NC64A, C. vulgaris, and C. sp. C-169) and marine environments (Prasinophyceae: M. pusilla, M. sp. RCC299,
O. sp. RCC809, O. tauri, and O. lucimarinus) each possessed a $b c h$ homolog. No CrtR- or bch-homolog was discovered in those algal strains from red algal secondary endosymbiotic event. Previous studies, however, demonstrated a xanthophyll cycle (zeaxanthin-antheraxanthinviolaxanthin), with violaxanthin the putative precursor of both diadinoxanthin and fucoxanthin in the diatom Phaeodactylum tricornutum [49,50], implying that this enzymatic reaction may therefore be catalyzed by other unrelated enzymes, such as LUT-like P450 proteins in those algal strains from Chromalveolates [51]. Therefore, isolation and characterization of novel enzymes involved in $\beta$ xanthophylls (zeaxanthin) biosynthesis in Chromalveolates are of going research.

Homologs of cyp $97 a$ and cyp 97 c genes are found in green algae, but absent in other algae, including red algae $(C$. merolae), Heterokontophyta (P. tricornutum, F. cylindrus and T. pseudonana), Haptophyta (E. huxleyi), Cryptophyta (G. theta), and Stramenopiles (A. anophagefferens). Interestingly, two cyp $97 a$ genes were predicted in C. reinhardtii and O. lucimarinus, indicating that lineage-specific gene duplications occurred during the evolution of these algae. Due to gene loss or the incompletely sequenced genome, only genes encoding CYP97B and CYP97C homologs and none gene encoding CYP97A homolog were predicted in B. natans CCMP2755. Moreover, none gene encoding CYP97A homolog has been discovered in Chlorella sp. NC64A. In this study, at least one copy of the gene encoding CYP97B was found to be widely distributed in most of algae, except for red algae, indicating that the originations of this gene is not due to secondary endosymbiosis.

\section{Domain structures of $\mathrm{BCH}$-type $\mathrm{CHYs}$ in algae}

$\beta$-Xanthophylls (zeaxanthin) are widely distributed in nature, and they are common in all photosynthetic eukaryotes, and many photosynthetic, or non-photosynthetic prokaryotes [26,52]. Non-heme/di-iron $\beta$-ring carotenoid hydroxylase $(\mathrm{BCH})$ has long been regarded as the only enzyme involved in zeaxanthin synthesis by hydroxylating $\beta$-carotene. Genes encoding $\beta$-carotene hydroxylases have been identified and characterized from bacteria [53,54], cyanobacteria [55], algae [41,42] and higher plants [34]. The $b c h$ genes of algae and higher plants encode the members of the fatty acid hydroxylase superfamily [PF04116] that also includes fatty acid and carotene hydroxylases and sterol desaturases. Members of this superfamily are integral membrane proteins and contain two copies of histidine-rich iron-binding motifs ( $\mathrm{HXXHH}$ ), needed for binding this cofactor with enzymes [34,56].

An alignment of the deduced amino acid sequences of CrtRs of cyanobacteria, and $\mathrm{BCHs}$ of red algae and green algae is displayed in Figure 2. There are few gaps in the alignment and a number of highly conserved regions. 
Table 1 Putative carotenoid hydroxylase genes identified in 18 algae genomes Gene locations (Locations are indicated by positions on either chromosomes or scaffolds) Protein length Types Chlorophyta_-Chlamydomonas reinhardtii

jgi|Chlre4|309780|kg.chromosome_8_\#_293_\#_ABQ59243.1

CYP97C

jgi|Chlre4|196742|DNE_DNE_gwH.55.10.1

jgi|Chlre4|196744|DNE_DNE_e_gwW.42.59.1

CYP97A

jgi|Ch|re4|283001|au.g1522_t1 CYP97B

jgi|Chlre4|164400|fgenesh2_kg.C_scaffold_40000013

\section{Chlorophyta_-Volvox carteri}

jgi|Volca1|83281|estExt_Genewise1Plus. C_520019

jgi|Volca1|65884|e_gw1.51.4.1

jgi|Volca1|44641|gw1.75.40.1

Chlorophyta_-Chlorella NC64A

jgi|ChINC64A_1|51247|fgenesh3_pg. C_scaffold_6000061

CYP97C

jgi|ChINC64A_1|138471||GS.gm_20_00220

jgi|ChINC64A_1|24463|e_gw1.13.95.1

Chlorophyta_CChlorella vulgaris

jgi|Chlvu1|60845|GG.C169_S07_00141

jgi|Chlvu1|24726|e_gw1.1.89.1

jgi|Chlvu1|26454|e_gw1.3.111.1

\section{Chlorophyta_-Coccomyxa sp c-169}

jgi|Coc_C169_1|63212|Genemark1.3995_g

CYP97C

jgi|Coc_C169_1|52277|estExt_fgenesh1_pm.C_10317

jgi|Coc_C169_1|12656|e_gw1.3.115.1

CYP97B

jgi|Coc_C169_1|30875|estExt_Genewise1Plus.C_160317

\section{Chlorophyta_-Ostreococcus lucimarinus}

jgi|Ost9901_3|33533|eugene.0900010237

CYP97C

jgi|Ost9901_3|47300|estExt_GenewiseEukaryote.C_Chr_130084

CYP97A

jgi|Ost9901_3|1824|gwEuk.21.62.1

CYP97A

jgi|Ost9901_3|29177|eugene.0100010571

CYP97B

jgi|Ost9901_3|18007|fgenesh1_pg.C_Chr_14000053

CYP97B

jgi|Ost9901_3|9013|gwEuk.10.453.1

$\mathrm{BCH}$

\section{Chlorophyta-O-Osetrococcus tauri}

jgi|Ostta4|1830|gw1.09.00.100.1

CYP97C

jgi|Ostta4|23029|estExt_fgenesh1_pm.C_Chr_13.00010043

CYP97A

jgi|Ostta4|27418|estExt_gwp_GeneWisePlus.C_Chr_01.00010469

CYP97B

jgi|Ostta4|23060|estExt_fgenesh1_pm.C_Chr_15.00010014

CYP97B

jgi|Ostta4|18835|e_gw1.08.00.85.1

OTHER

jgi|Ostta4|5089|gw1.10.00.289.1

$\mathrm{BCH}$

\section{Chlorophyta-O Osetrococcus RCC809}

jgi|OstRCC809_1|1644|gw1.3.120.1 
Table 1 Putative carotenoid hydroxylase genes identified in 18 algae genomes (Continued)

\begin{tabular}{|c|c|c|}
\hline jgi|OstRCC809_1|53931|estExt_Genewise1.C_21287 & 525 & CYP97B \\
\hline jgi|OstRCC809_1|87721|eugene1.0000120100 & 576 & CYP97B \\
\hline jgi|OstRCC809_1|16226|gw1.3.1299.1 & 153 & $\mathrm{BCH}$ \\
\hline \multicolumn{3}{|l|}{ Chlorophyta_-Micromonas pusilla } \\
\hline jgi|MicpuC2|32152 & 550 & CYP97C \\
\hline jgi|MicpuC2|26780 & 580 & CYP97A \\
\hline jgi|MicpuC2|22138 & 530 & CYP97B \\
\hline jgi|MicpuC2|57732 & 526 & OTHER \\
\hline jgi|MicpuC2|11104 & 230 & $\mathrm{BCH}$ \\
\hline \multicolumn{3}{|l|}{ Chlorophyta_-Micromonas sp. RCC299 } \\
\hline jgi|MicpuN2|95887|estExt_Genewise2Plus.C_Chr_140254 & 542 & CYP97C \\
\hline jgi|MicpuN2|83128|e_gw2.06.152.1 & 525 & CYP97A \\
\hline jgi|MicpuN2|96121|estExt_Genewise2Plus.C_Chr_160324 & 539 & CYP97B \\
\hline jgi|MicpuN2|88940|e_gw2.16.55.1 & 574 & CYP97B \\
\hline jgi|MicpuN2|76186|gw2.07.573.1 & 232 & $\mathrm{BCH}$ \\
\hline \multicolumn{3}{|l|}{ Rhodophyta_-Cyanidioschyzon merolae } \\
\hline gnl|CMER|CMV041C [pt] beta-carotene hydroxylase & 259 & CRTR \\
\hline \multicolumn{3}{|l|}{ Bacillariophyta_-Thalassiosira pseudonana } \\
\hline jgi|Thaps3|36235|e_gw1.9.19.1 & 667 & CYP97B \\
\hline jgi|Thaps3|264039|thaps1_ua_kg.chr_13000087 & 547 & CYP97B \\
\hline \multicolumn{3}{|l|}{ Bacillariophyta_—Phaeodactylum tricornutum } \\
\hline jgi|Phatr2|26422|estExt_Genewise1.C_chr_50056 & 770 & CYP97B \\
\hline jgi|Phatr2|16586|e_gw1.27.30.1 & 539 & CYP97B \\
\hline \multicolumn{3}{|l|}{ Bacillariophyta_—Fragilariopsis cylindrus } \\
\hline jgi|Fracy1|169705|estExt_Genewise1.C_61231 & 528 & CYP97B \\
\hline jgi|Fracy1|170430|estExt_Genewise1.C_71165 & 614 & CYP97B \\
\hline \multicolumn{3}{|l|}{ Haptophyceae_-Emiliania huxleyi } \\
\hline jgi|Emihu1|463287|estExtDG_fgeneshEH_pg.C_230139 & 618 & CYP97B \\
\hline \multicolumn{3}{|l|}{ Cryptophyta_-Guillardia theta } \\
\hline jgi|Guith1|114743|au.78_g15845 & 582 & CYP97B \\
\hline jgi|Guith1|88554|estExt_Genewise1Plus.C_630014 & 492 & CYP97B \\
\hline jgi|Guith1|158065|fgenesh2_pm.48_\#_5 & 499 & CYP97B \\
\hline \multicolumn{3}{|l|}{ Cercozoa_—Bigelowiella natans CCMP2755 } \\
\hline jgi|Bigna1|39488|e_gw1.33.13.1 & 474 & CYP97C \\
\hline jgi|Bigna1|52980|estExt_Genewise1Plus.C_140020 & 545 & CYP97B \\
\hline \multicolumn{3}{|l|}{ Stramenopiles_-Aureococcus anophagefferens } \\
\hline jgi|Auran1|19592 & 528 & CYP97B \\
\hline jgi|Auran1|34662 & 432 & CYP97B \\
\hline
\end{tabular}

OTHER indicates two genes belonged to no one subfamily of CYP97.

Four transmembrane segments and four histidine-rich boxes (HKXLWH, HXSHH, HDGLVH, and HXXHH) are distributed among the amino acid residues of $\mathrm{BCHs}$ of green algae (Figure 2). It is interesting that the first transmembrane segment was absent in two BCHs of green algae O. sp. RCC809 and O. lucimarinus, and replaced instead by another histidine-rich box in the N-terminal (HKHLWH). Three conserved histidine-rich boxes (HDASHXXAH, HXQHHXX, and HLIHH) and five transmembrane segments are generally well-conserved between red algae and cyanobacteria, indicating that red algae acquired this gene from cyanobacteria during 


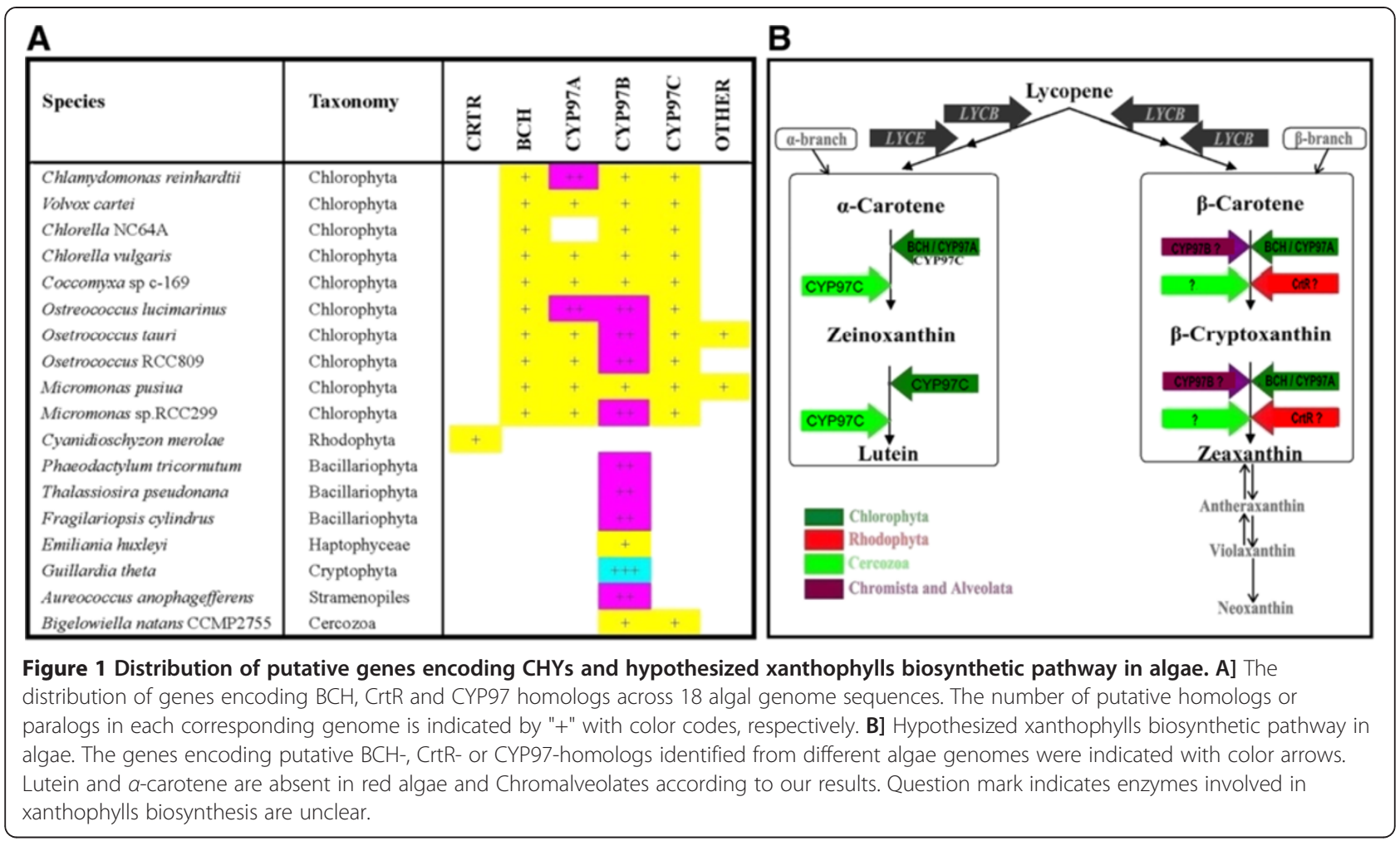

primary endosymbiosis (Figure 2). However, previous studies have demonstrated that the protein sequence of the C. merolae $\mathrm{BCH}$ (CrtR-type) is truncated at the $\mathrm{N}$ terminus by at least 22 amino acids relative to the sequences of all cyanobacterial CrtRs, and that the activity of this encoded enzyme could not be shown when expressed in E. coli $[57,58]$. Two HXXHH boxes are widespread across the $\mathrm{BCHs}$ of algae and CrtRs of cyanobacteria, indicating that their catalytic mechanisms are highly similar in these proteins $[34,56]$.

\section{Origins and evolution of $\mathrm{BCHs}$ in algae}

$\beta$-Carotene hydroxylation in different organisms is primarily carried out by members of three subfamilies of the non-heme/di-iron monooxygenase superfamily: $\mathrm{BCH}$-type enzyme of green algae and higher plants; $\mathrm{BCH}$-type enzyme of non-photosynthetic bacteria; and CrtR-type enzyme of cyanobacteria. The former shares sequence homology with non-photosynthetic bacterial-type enzyme, and the latter (CrtR-type) is more closely related to bacterial carotenoid ketolases [59]. Identification of plant-like $\mathrm{BCHs}$ in green algae and cyanobacteria-like CrtRs in red algae is the key indicating complicated origination of $\mathrm{BCH}$ in algae, for which we constructed a phylogenetic tree of CrtRs of cyanobacteria and $\mathrm{BCHs}$ of green algae, higher plants, and bacteria (Figure 3) (Additional file 3: Table S2).

As shown in Figure 3, difference between CrtRs of cyanobacteria and $\mathrm{BCHs}$ of algae, bacteria, and higher plants is very clear. Genes encoding $\mathrm{BCH}$ from bacteria, green algae, and higher plants build a monophyletic group (Bootstrap [BS]: 100\%), and the phylogenetic relationship present here indicates that they share a common ancestor and strongly supports a non-cyanobacterial origin. In contrast to the apparently widespread retention of $\mathrm{BCH}$ paralogs in higher plants, the gene encoding $\mathrm{BCH}$ is a single copy in each algal genome sequences (Figure 3). In higher plants, duplication of the ancestral $b c h$ gene took place most probably via whole-genome or segmental genome duplication [26] and the duplicates seem to have functionally diverged primarily at the gene expression level [60-62]. It is worth mentioned, however, that the gene encoding $\mathrm{BCH}$ (CrtR-type) of red algae forms another monophyletic group (BS: 97\%) with CrtRs of cyanobacteria, suggesting that the red alga obtained this gene via the primary endosymbiotic event from cyanobacteria and retained it over the course of evolution persistently. However, the activity of this encoded enzyme was not demonstrated when expressed in E. coli $[57,58]$. Therefore, hydroxylation of $\beta$-carotene in red algae remains unclear, calling for more red-algal genomes sequences to elucidate. In this study, no $\mathrm{BCH}$ - or CrtR-type CHY-encoding gene was discovered in algae of Chromalveolates, except for a partial sequence of bch-type gene in the genome sequences of $T$. pseudonana. This result is consistent with observations from a previous study, in which only a partial sequence of $\mathrm{BCH}$-type gene was found in the 


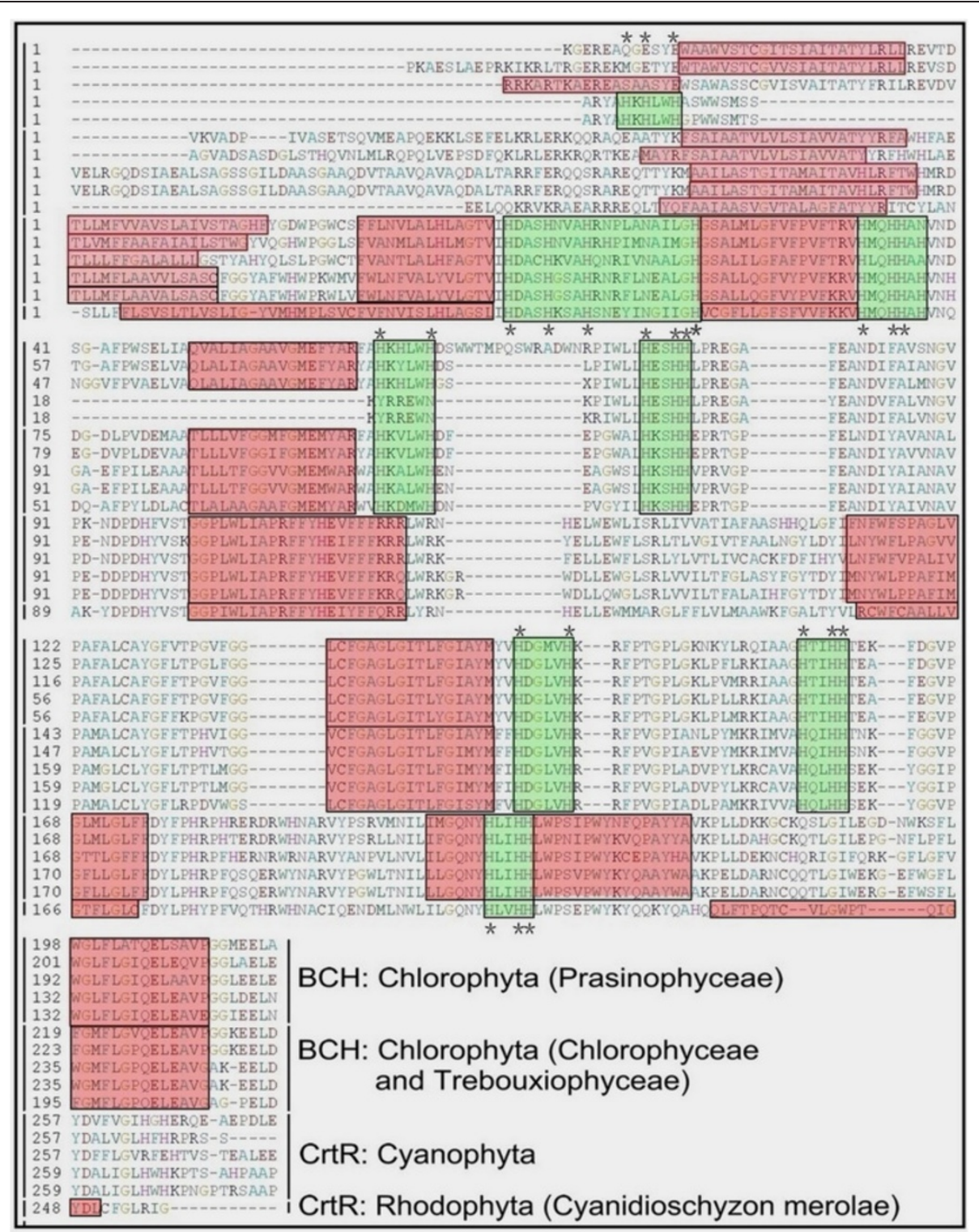

Figure 2 Domain structure of $\mathbf{B C H}$ from green algae and CrtR from cyanobacteria and red algae. Ten BCH-type CHYs from green algae, one CrtR-type CHY from red algae and five CrtRs from cyanobacteria are included. A partial protein sequence (position: 110-400) has been selected for domain structure analysis. The predicted trans-membrane segments are shaded in red. The histidine boxes are shaded in green and Black stars indicate the positions of conserved histidine residues. Ten $\mathrm{BCH}$-type $\mathrm{CHYs}$ from green algae includes Prasionphyceae [Micromonas pusilla, Micromonas sp. RCC299, Ostreococcus sp. RCC809, Ostreococcus tauri, and Ostreococcus lucimarinus]. One CrtR-type CHY from the red alga is Cyanidioschyzon merolae. The information of $\mathrm{BCH}$ genes from algae is as in Table 1. Five CrtRs from cyanobacteria includes Synechococcus sp. JA-2-3B'a (2-13) [Cyanobase: CYB_0102], Synechococcus sp. JA-3-3Ab [Cyanobase: CYA_1931], Cyanothece sp. PCC 7425 [Cyanobase:

Cyan7425_1008], Acaryochloris marina MBIC11017 [Cyanobase: AM1_3637] and Thermosynechococcus elongatus BP-1 [Cyanobase: tr1900].

genome sequences of $T$. pseudonana and no one was detected from the $P$. tricornutum genome [51]. Unfortunately, the enzymatic activity of this putative carotene hydroxylases $(\mathrm{BCH})$ from $T$. pseudonana has not yet been reported anywhere to the best knowledge of authors. Therefore, the hydroxylation of $\beta$-carotene in Chromalveolates remains unknown, resulting from genomes of the few organisms currently sequenced lack entire hydroxylation families and, enzymatic activity of putative carotene hydroxylases has not yet been reported at present [51,57,63-65].

As mentioned above, it is difficult to fully understand the evolution of $\beta$-xanthophylls biosynthesis with limited available data, but some hypotheses were proposed based on the tree topology. Firstly, it is presumed that CrtR-type $\mathrm{CHY}$ was originated in all algae as the result of primary endosymbiosis. In such a scenario, CrtR-type $\mathrm{CHY}$ proteins were lost in extant green algae and higher 


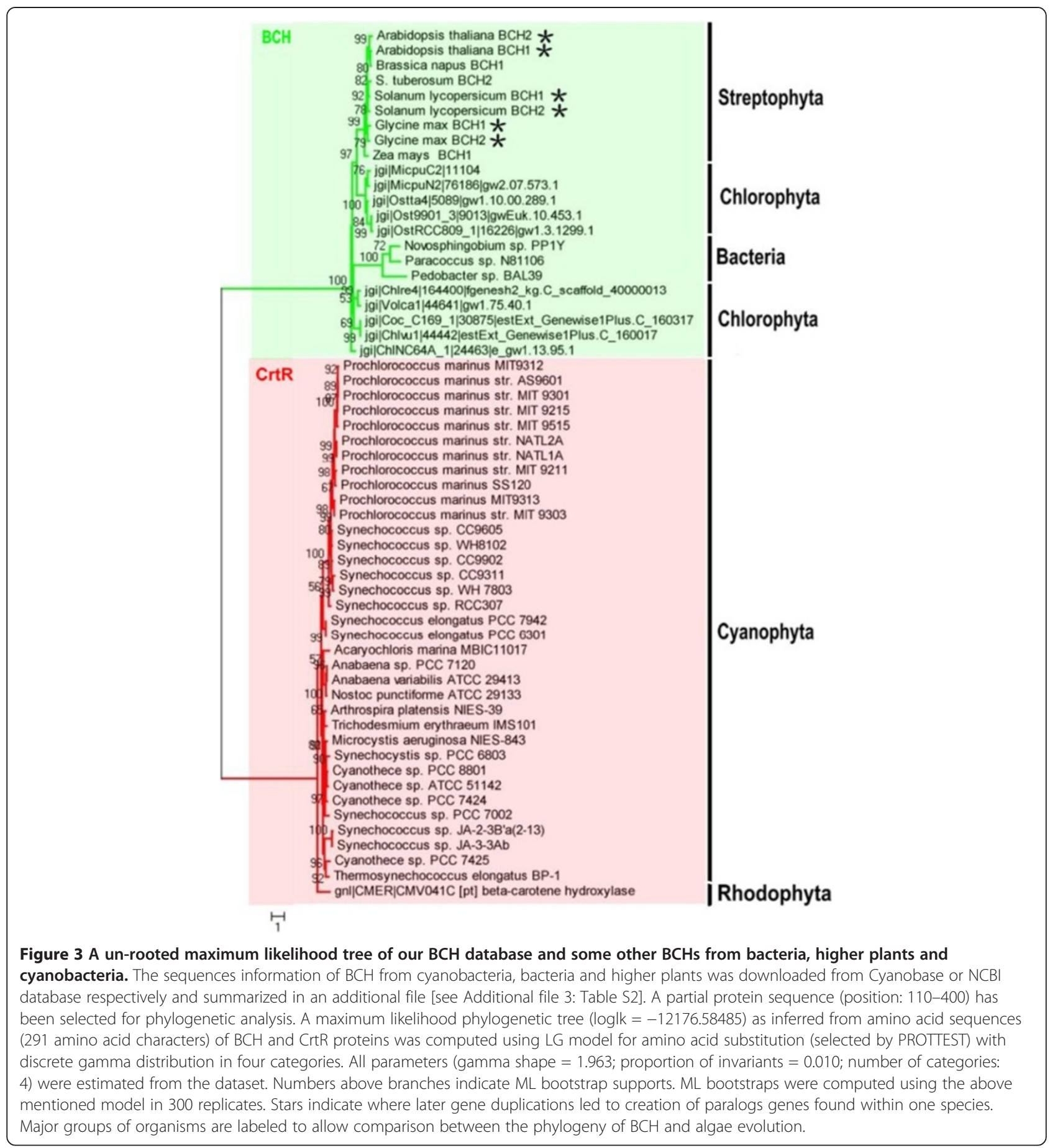

plants, and these organisms acquiring another $\mathrm{BCH}$ from bacteria by lateral gene transfer or from the host during the primary endosymbiosis event. Secondly, red algae have CrtR-type CHY-encoding gene, while Chromalveolates do not have them due possibly to gene lost during the secondary endosymbiosis event. In addition, $\mathrm{BCH}$-type $\mathrm{CHYs}$ are also absent in Chromalveolates, which may be resulted from the replacement of algal CrtR- and $\mathrm{BCH}$-type $\mathrm{CHYs}$ by another unidentified novel lineage-specific
$\mathrm{CHY}$ in strains that derived from the red algal secondary endosymbiosis. Previous studies of the diatom $P$. tricornutum [43], however, have demonstrated the presence of a xanthophyll cycle (zeaxanthin-antheraxanthinviolaxanthin), with violaxanthin the putative precursor of both diadinoxanthin and fucoxanthin, implying this enzymatic reaction may therefore be catalyzed by other unrelated enzymes, such as LUT-like P450 proteins [51]. Therefore, we speculate that the bch genes in 
Chromalveolates have not been under a strong pressure of natural selection like in green algae and higher plant lineages, and they may have instead evolved different ways for $\beta$-xanthophylls biosynthesis.

\section{Domain structures of CYP97s in algae}

Cytochrome P450s are defined by the $450 \mathrm{~nm}$ light absorption of their heme cofactors. They oxidize various arrays of metabolic intermediates and environmental compounds [66], and participate in many primary, secondary, and xenobiotic metabolic reactions [67]. The CYP97-encoding genes are members of P450s family. These members share a common catalytic center in heme by iron coordination to the thiolate of a conserved cysteine [68]. Despite low sequence identity at amino acid level, P450s display a common overall topology in threedimensional folding pattern $[69,70]$. Genes encoding members of CYP97 family have been isolated and functionally investigated from higher plants $[8,26,33,37,59,71,72]$. There are three CYP97 family members (CYP97A3, CYP97B3, and CYP97C1) in Arabidopsis genome, of which CYP97A3 and CYP97C1 are predicted to be chloroplast-targeted $[59,73]$. The localization of predicted chloroplast CYP97A/ $\mathrm{C}$ from algae and higher plants is coincident with the sub-cellular of carotenoids biosynthesis. To further reveal domain structure characteristics of CYP97 proteins, an alignment was constructed using selected CYP97A/B/C protein sequences from different algae (Figure 4) and an additional file shows this in more detail [see Additional file 4: Figure S1].

The alignment (Figure 4) reveals 11 domains in strongly conserved amino acid sequences from all CYP97 protein sequences. All conserved domains are highly similar between CYP97As and CYP97Cs of green algae except for CD1 (QPVFVPLYKLPLXYG) (Figure 4), indicating that they share a common ancestor. In addition, the conserved domains in CYP97Bs of distinct algae phyla are also similar, implying that this gene occurred before the formation of extant diverse algal groups. Degenerate primers for cloning genes encoding CYP97 homologs were designed from these conserved amino acid residues. P450s catalytic motifs were found as expected in all CYP97A/B/C protein sequences including I-helix involved in oxygen binding (CD6 in CYP97B and CD5 in CYP97A and CYP97C), an ERR triad (CD7 in CYP97A/B/C) responsible for locking the heme pockets into position and assuring stabilization of the conserved core structure, and CD10 associated with the conserved heme-binding cysteine. In addition, many amino acids were conserved in each CYP97 subfamily such as CD4 (LLRFLVDXR) and CD9 (LYPXE) were conserved in CYP97B subfamily only. The conserved domains associated with active sites suggest that these protein sequences are members of the P450 family, and that conserved domains within each CYP97 subfamily proteins are responsible for the specificity with respect to the $\beta$ - or $\varepsilon$-ring of different carotenes.

\section{Origins and evolution of CYP97s in algae}

Production of $\alpha$-xanthophylls (lutein) in higher plants requires four reaction steps: $\beta$ - and $\varepsilon$-ring formation from lycopene by the action of $\beta$ - and $\varepsilon$-cyclases (LYCB and LYCE); and subsequently, hydroxylation of each ring of $\alpha$-carotene by $\beta$ - and $\varepsilon$-ring hydroxylases (CYP97A and CYP97C) [26]. Ample evidence shows that genes encoding CYP97A and CYP97C homologs are only present in green algae and higher plants, but not in Chromalveolates, indicating that $\alpha$-xanthophylls biosynthesis occurs only in green algae and higher plant lineages. In our results, this biosynthetic pathway is also absent in red algae. In contrast to a previous study postulated that synthesis of $\alpha$-xanthophylls occurs in only a few lineages of photosynthetic eukaryotes, namely, some red algae, all green algae, and higher plants [26]. An insufficient number of red algal species (only a red alga used in this study) may be responsible for these different conclusions. In addition, our previous and other studies have demonstrated that $\beta$-cyclases (LYCBs) genes are widely distributed in nature, and $\varepsilon$-cyclase (LYCEs) genes were identified only in green algae, higher plants, and some cyanobacteria (e.g. Prochlorococcus marinus MED4). LYCE genes seems to come from $\beta$-cyclases by gene duplication and subsequently functional divergence [30,57,74-77], which indirectly manifests that $\alpha$-xanthophylls are synthesized only in green algae and higher plants. No CYP97A and CYP97C homologs are detected in algal genome sequences from Chromalveolates, and these algae cannot synthesize $\alpha$-xanthophylls (lutein), which is consistent with previous studies [51].

At present, no CYP97 protein homolog has been found in cyanobacteria, suggesting that these proteins are an ancient eukaryotic innovation. All three CYP97 subfamilies are represented in Arabidopsis and other land plants, often in a single copy per subfamily, indicating their critical functions [26]. In contrast, some paralogs genes encoding CYP97A in a few green algae and CYP97B in most algae, especially in Chromalveolates, indicating recent gene duplication events occurred. For deep understanding the internal phylogenetic relationships among CYP97 proteins from higher plants and green algae, we constructed a maximum likelihood tree using our CYP97 database and some other CYP97s from higher plants (Figure 5). CYP86A1 from Arabidopsis thaliana was selected as outgroup because its substrates, fatty acids with chain lengths from $\mathrm{C}_{12}$ to $\mathrm{C}_{18}$ [78] are mostly molecules similar to carotenoids, and the CYP86 clade is the most closely related to the CYP97 clade [26].

As it shows in maximum likelihood tree, CYP97 proteins constitute three distinct monophyletic groups: CYP97A, 


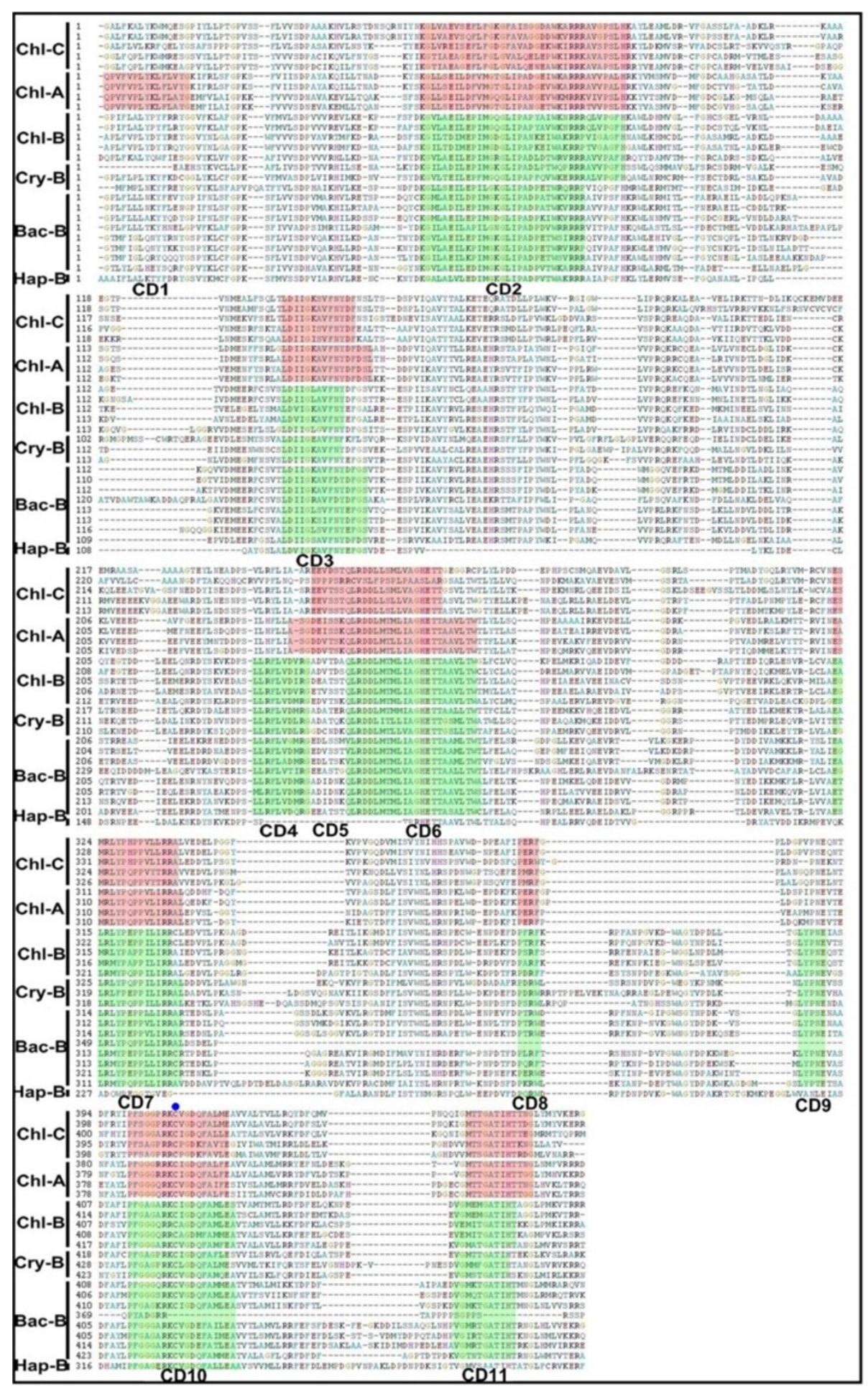

Figure 4 (See legend on next page.) 
(See figure on previous page.)

Figure 4 Domain structure of CYP97 from algae. A partial protein sequence (position: 272-926) has been selected for domain structure analysis. The red shades indicated conserved amino acid residues in CYP97A and CYP97C homologs from green algae. The green shades indicated conserved amino acid residues in all CYP97B homologs from all algae. The P450s active site components were found in the amino acid sequences of all CYP97A/B/C, including /-helix involved in oxygen binding (CD6 in CYP97B and CD5 in CYP97A and CYP97C), ERR triad (CD7 in CYP97A/B/C) involved in locking the heme pockets into position and to assure stabilization of the conserved core structure, and CD10 involved in heme binding and a conserved cysteine (the circle with blue color). The abbreviations used are: Chl-C, CYP97C from Chlorophyta C. reinhardtii, V. carteri, M. sp. RCC299, O. RCC809, and Cercozoa B. natans CCMP2755; Chl-A, CYP97A from Chlorophyta C. reinhardtii, V. carteri, M. sp. RCC299, and O. RCC809; Chl-B, CYP97B from Chlorophyta M. sp. RCC299, O. RCC809, and V. carteri; Cry-B, CYP97B from Cryptophyta G. theta; Bac-B, CYP97B from Bacillariophyta T. pseudonana, P. tricornutum, F. cylindrus, and Stramenopiles A. anophagefferens; Hap-B, CYP97B from E. huxleyi. The information of BCH genes from algae is as in Table 1.

CYP97B, and CYP97C. Two of these groups (CYP97A and CYP97C) form a sister group that are composed of proteins from green algae and higher plants. CYP97A and CYP97C sequences in Chromalveolates, including Heterokontophyta (P. tricornutum, F. cylindrus, and T. pseudonana), Haptophyta (E. huxleyi), Cryptophyta (G. theta), and Stramenopiles (A. anophagefferens) are absent in the cluster. Genes encoding CYP97B homologs from all organisms form a monophyletic group (BS: 92\%). Surprisingly, algal CYP97Bs from Chromalveolates and green algae (Prasinophyceae) build another monophyletic group (BS: 95\%). This phylogenetic relationship and the lack of CYP97B homologs in C. merolae point to a "green" origin of this gene in Chromalveolates, similar to the origin and evolution of hydroxypyruvate reductase [79]. Alternatively, this gene represents early eukaryotic innovations in the Plantae although lacking CYP97B homolog in $C$. merolae. Further investigations are needed to support this hypothesis.

The topology of the phylogenetic tree shows that the CYP97B is an ancient gene emerging before the divergence of extant algae groups during evolution, and represents an ancient eukaryotic innovation. Our results indicate that CYP97A is evolutionarily more closely to CYP97C than to CYP97B (Figure 5). Therefore, we believe that the CYP97A and CYP97C genes were originated by gene duplication before the split between green algae and higher plants, and were subjected to purifying selection in a lineage-specific fashion (Figure 5). Alternatively, these genes may have been originally present in all algae, and then they were lost gradully from red algae and Chromalveolates. This scenario is similarity to that of Arabidopsis whose evolution and functional divergence of two duplicate gene pairs, CYP97A3/C1 and BCH1/2 involved in carotenoid hydroxylation occurred [26]. Additional lineage- or organism-specific gene duplications have occurred during the evolution of CYP97A in green algae (C. reinhardtii and O. lucimarinus), and CYP97B in most algae, except for $C$. reinhardtii, $C$. sp. NC64A, $C$. vulgaris, C. sp. C-169, V. carteri, M. pusilla, E. huxleyi, and $B$. natans CCMP2755. Duplication and subsequent functional divergence of genes have been recognized increasingly as an important mechanism of evolution [80-83].

\section{Isolation and characterization of cyp97 genes from $\mathrm{H}$. pluvialis}

To further study genes encoding CYP97 homologs in green algae, three full-length cDNA sequences of cyp 97 homologs, including Haecyp 97a, Haecyp97b, and Haecyp 97c [84] were isolated from commercial green alga $H$. pluvialis strain Flotow 1844. Briefly, 1,017- and 984-bp cDNA fragments encoding HaeCYP97A/B were generated by RTPCR with degenerate primers [see Additional file 5: Table S3]. The Haecyp $97 a$ fragment shared $77 \%$ sequence similarity with cyp $97 a 5$ gene of $C$. reinhardtii and $74 \%$ similarity with cyp97a3 gene of $A$. thaliana. The Haecyp $97 b$ fragment shared $61 \%$ sequence similarity with $A$. thaliana cyp $97 b 3$ gene. The results indicate that two partial putative cyp $97 a / b$ genes were isolated from H. pluvialis. Gene-specific primers were then designed to obtain full-length sequences of Haecyp $97 a / b$ using RACE methods. Information regarding the full-length sequences of Haecyp $97 a / b / c$ is summarized in Table 2 . The full-length cDNA sequences of Haecyp 97 a comprised 1,872-bp with an open reading frame (ORF) of 1,593-bp encoding a 530 amino acid protein, and it was flanked by a 159-bp of 5 '-untranslated region (UTR) and a 120-bp of 3 '-UTR including the poly-A tail [see Additional file 6: Figure S2]. The deduced protein had a calculated molecular weight of $59.03 \mathrm{kDa}$ and a predicted isoelectric point (pI) of 7.81. The full-length cDNA sequences of Haecyp $97 b$ contained an ORF of 1,620-bp, a 2-bp 5'-UTR, and a 249-bp 3'-UTR, and it encoded a new putative carotenoid hydroxylase protein of 539 amino acid protein with a deduced molecular weight of $58.72 \mathrm{kDa}$ and pI of 6.26 [see Additional file 7: Figure S3]. Sequence analysis revealed that the cloned Haecyp 97 c cDNA was 1,995-bp in length, and contained a 1,620-bp ORF, a 46-bp 5' -UTR, and a 329-bp 3 '-UTR in characteristic of a poly (A) tail. An ATG translation initiation codon was identified in the 46-bp terminal sequence (47-49 bp), and a TAA termination codon was found in the $1,620 \mathrm{bp}$ downstream of the initiation site [see Additional file 8: Figure S4].

The ChloroP and TargetP servers $[85,86]$ were used to predict the sub-cellular location of the respective deduced proteins from isolated genes (Table 2). The results 


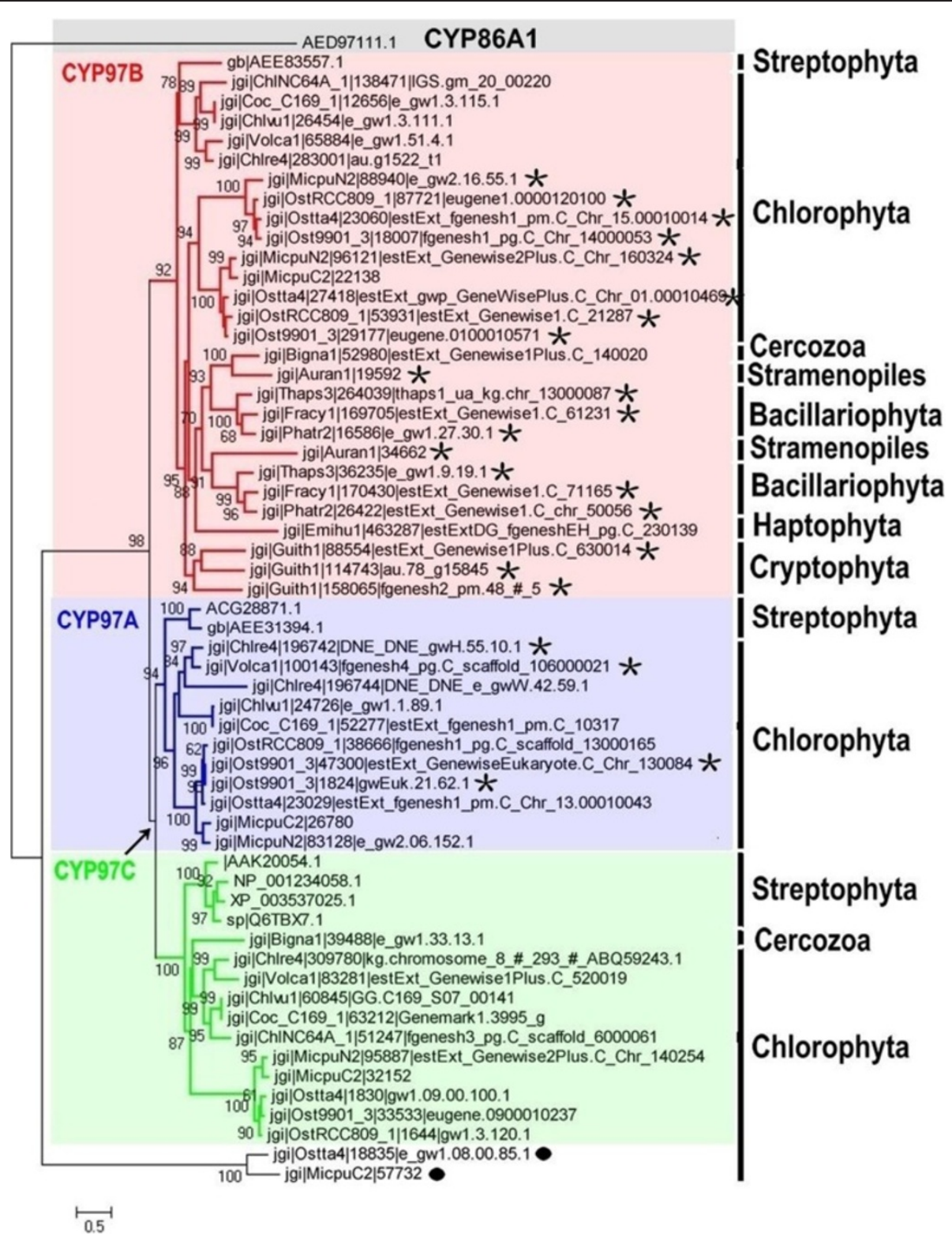

Figure 5 A maximum likelihood tree of our CYP97 database and some other CYP97s from higher plants. The sequences information of CYP97s from higher plants was downloaded from NCBI database and summarized as follow: Arabidopsis thaliana [GenBank: CYP97A3, gb| AEE31394.1, CYP97B3, gb|AEE83557.1, CYP97C1, sp|Q6TBX7.1 and CYP86A, AED97111.1], Zea mays [GenBank: CYP97A16, ACG28871.1], Glycine max [GenBank: carotene epsilon-monooxygenase, XP_003537025.1], Solanum lycopersicum [GenBank: CYP97C11, NP_001234058.1] and Oryza sativa Japonica Group [GenBank: carotene epsilon-monooxygenase, AAK20054.1]. A partial protein sequence (position: 272-926) has been selected for phylogenetic analysis. A maximum likelihood phylogenetic tree (loglk $=-27808.68723)$ as inferred from amino acid sequences (655 amino acid characters) of CYP97 proteins was computed using LG model for amino acid substitution (selected by PROTTEST) with discrete gamma distribution in four categories. All parameters (gamma shape $=1.924$; proportion of invariants $=0.011$; number of categories: 4 ) were estimated from the dataset. Numbers above branches indicate ML bootstrap supports. ML bootstraps were computed using the above mentioned model in 300 replicates. The arrow indicates an ancient gene duplication event creating CYP97A/C, respectively. Stars indicate where later gene duplications led to creation of paralogs genes found within one species. Black circle indicate two genes belonged to no one subfamily of CYP97. Major groups of organisms are labeled to allow comparison between the phylogeny of CYP97A/B/C and algae evolution.

indicate that CYP97A and CYP97C in H. pluvialis are probably located in the chloroplast, same as in higher plants Arabidopsis thaliana [59,73]. We also calculated degrees of identity and similarity between predicted amino acid sequences of each isolated CYP97 and corresponding CYP97s of other eukaryotes (Table 3). Amino acid sequences from each of the isolated CYP97 genes shared high similarities (72\%-76\%) with known eukaryotic 
Table 2 Listed of three full-length of HaeCYP97A/B/C from Haematococcus pluvialis

\begin{tabular}{|c|c|c|c|c|c|c|c|c|c|}
\hline & mRNA (bp) & 5'UTR (bp) & CDS (bp) & 3'UTR (bp) & Protein (aa) & MW (kDa) & pl & TM & SL \\
\hline HaeCYP97A & 1872 & $1-159$ & $160-1752$ & $1753-1872$ & 530 & 59.03 & 7.81 & - & chl \\
\hline HaeCYP97B & 1871 & $1-2$ & 3-1622 & 1623-1871 & 539 & 58.72 & 6.26 & - & nd \\
\hline HaeCYP97C & 1995 & $1-46$ & 47-1666 & 1667-1995 & 539 & 58.71 & 7.94 & - & $\mathrm{chl}$ \\
\hline
\end{tabular}

Abbreviations: 5'UTR 5'-untranslated region, 3'UTR 3'-untranslated region, CDS coding sequences, Mw molecular weight, $p$ isoelectric point, TM Transmembrane regions, SL subcellular localization, $b p$ base pair, chl chloroplast.

proteins, indicating that the three novel cyp 97 genes of green alga $H$. pluvialis Flotow 1844 has been successfully identified using RT-PCR with degenerate primers designed from conserved motifs and RACE methods. To our knowledge, this is the first time these three cyp 97 gene homologs have been isolated from commercial green algae.

Expression analysis of four chy genes in $H$. pluvialis under high light stress

Studies showed that high light (HL) effectively induces carotenoid biosynthesis-related gene expression and astaxanthin accumulation in $H$. pluvialis $[47,48]$, indicating that light plays an important role in controlling green algal carotenoid biosynthesis. Although the regulatory role of light in the expression of nuclear-encoded plastid-targeted proteins has been studied for decades in green algae and higher plants, elucidation of effects of light on transcriptional levels of three novel cyp 97 genes in green algae is still in its infancy. In a previous study on diatom P. tricornutum, a blue-light library was found to be the most enriched in carotenogenesis-related ESTs [51]. For more detail in the transcriptional regulatory role of light on green algae, we studied the gene transcriptional expression profiles of bch, Haecyp97a, Haecyp97b, and Haecyp $97 c$ genes in response to white and blue HL conditions.

As shown in Figure 6, transcriptional levels of four chy genes (bch, Haecyp97a, Haecyp97b, and Haecyp97c) were increased throughout the course of HL illumination treatments. Starting from relatively low levels, $b c h$ expression level was slowly increased under blue HL treatment and reached a maximum transcriptional level at $24 \mathrm{~h}$ exposure that was 5.0 -fold higher than that of the control. It then declined sharply after $72 \mathrm{~h}$ of exposure (Figure 6A). A similar, although less pronounced trend was observed under white HL: the highest $b c h$ transcriptional level occurred at $54 \mathrm{~h}$ of exposure, with 3.3-fold higher compared with the control. Our previous study has demonstrated that zeaxanthin concentrations under blue and white HL treatments were increased markedly and reached their highest levels at $34 \mathrm{~h}$ (blue) and $48 \mathrm{~h}$ (white) of exposure [84], which is listed in Figure S5 for details [see Additional file 9: Figure S5]. $\mathrm{BCH}$ is the mainly enzyme catalyzing the hydroxylation of $\beta$-carotene, which produces zeaxanthin associated with the xanthophylls cycle $[40,87]$. The contradiction between low bch expression levels and marked increase of zeaxanthin concentration during the early stage of treatments was explained by the fact that when photoprotection is required, violaxanthin is rapidly converted via antheraxanthin to zeaxanthin by violaxanthin de-epoxidase [88]. The astaxanthin concentration under blue or white HL stress was higher than that of the control, reaching a maximum level at $34 \mathrm{~h}$ and $54 \mathrm{~h}$ of exposure, respectively [84], which is listed in Figure S5 for details [see Additional file 9: Figure S5]. Accumulation of astaxanthin may be responsible for the rapidly increasing levels of $b c h$ and sharply decreasing concentrations of zeaxanthin at later treatment stages. Previous studies have demonstrated that $b c h$ and $b k t$ are the main genes involved in astaxanthin biosynthesis [89].

Expression levels of Haecyp97a, Haecyp97b, and Haecyp97c began with a slight decrease followed by a dramatic increase (Figure 6B-D). Strongly steady increase in transcriptional levels of Haecyp $97 a$ and Haecyp $97 b$ were observed during $4-28 \mathrm{~h}$ of both HL (white and blue)

Table 3 The degree of identity and similar between the predicted amino acid sequences for each isolated CYP97 genes and corresponding CYP97 from other eukaryotes

\begin{tabular}{|c|c|c|c|c|c|c|}
\hline & HaeCYP97A & HaeCYP97B & HaeCYP97C & AthCYP97A3 & AthCYP97B3 & AthCYP97C1 \\
\hline HaeCYP97A & $100 / 100$ & $51 / 66$ & $46 / 64$ & $57 / 72$ & $48 / 64$ & $50 / 70$ \\
\hline HaeCYP97B & & $100 / 100$ & $43 / 59$ & $48 / 63$ & $61 / 76$ & $44 / 60$ \\
\hline HaeCYP97C & & & $100 / 100$ & $46 / 62$ & $39 / 56$ & $61 / 72$ \\
\hline AthCYP97A3 & & & & $100 / 100$ & $40 / 65$ & $53 / 72$ \\
\hline AthCYP97B3 & & & & & $100 / 100$ & $43 / 60$ \\
\hline AthCYP97C1 & & & & & & $100 / 100$ \\
\hline
\end{tabular}

Note: AthCYP97A3 [GenBank: NP_564384.1], AthCYP97B3 [GenBank: NP_193247.2] and AthCYP97C1 [GenBank: NP_190881.2] were downloaded from National Center for Biotechnology Information GenBank database, respectively. The data was present by the form of identity/similarity. 


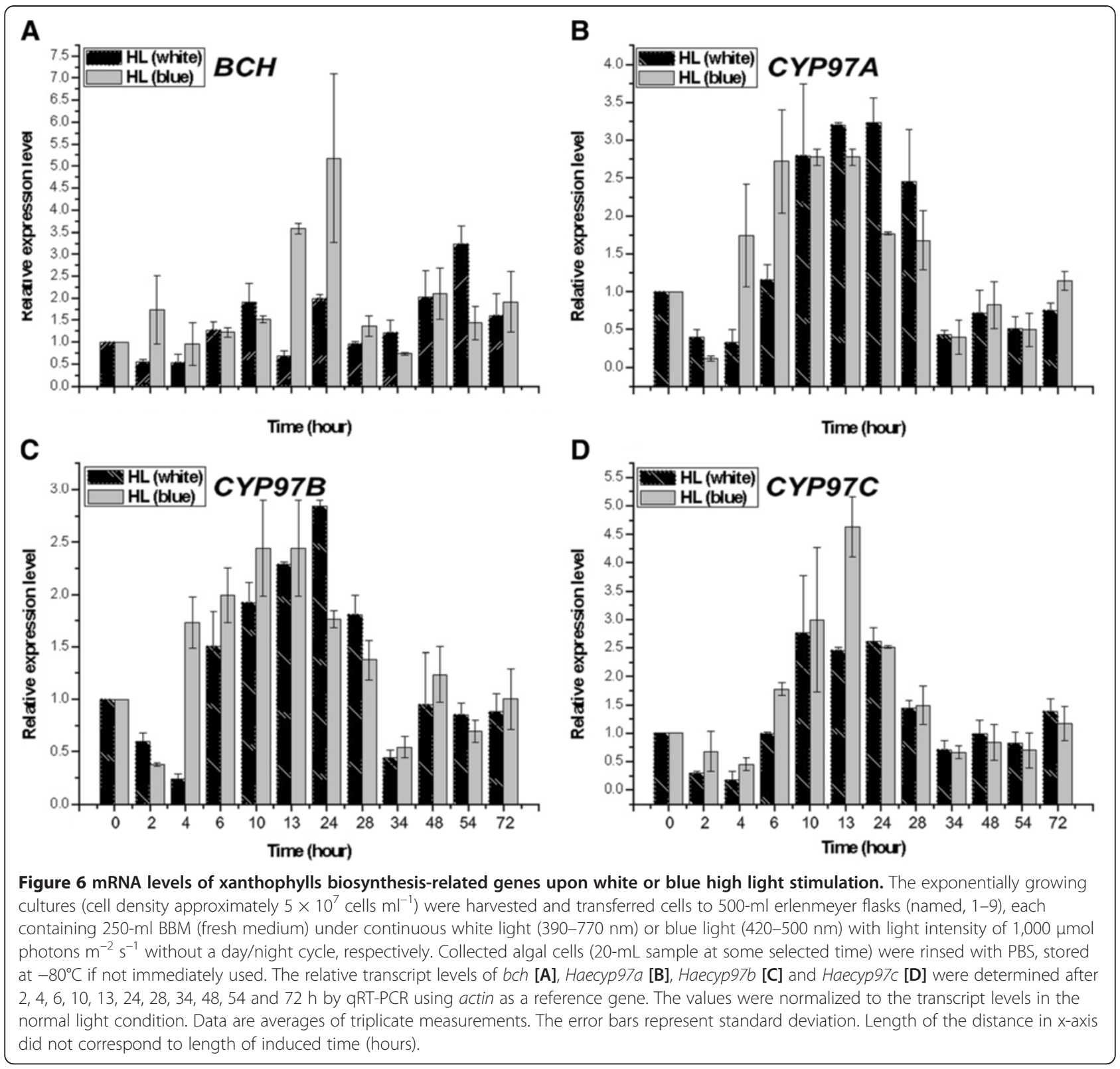

treatments. Then, they dropped sharply after $28 \mathrm{~h}$ of exposure. It is also intriguing that different conditions had various impacts on Haecyp $97 a$ and Haecyp $97 b$ mRNA levels. For instance, transcriptional levels of Haecyp $97 a$ and Haecyp $97 b$ under blue HL were higher than that of under white $\mathrm{HL}$ during $4-10 \mathrm{~h}$ of exposure, and the contrary tendency was observed during $13-28 \mathrm{~h}$ of exposure (Figure 6B and $\mathrm{C}$ ). The maximum transcriptional levels of Haecyp97c under both the blue and white HL treatments occurred at hour 13 and 10, and transcriptional levels were 4.5- and 2.8-fold higher than that of control, respectively (Figure 6D). Blue HL appeared to have stronger effects on Haecyp $97 c$ transcriptional level than that of white HL (Figure 6D).
Studies on mutant of higher plants showed that CYP97A3 and CYP97C1 are the enzymes primarily responsible for catalyzing hydroxylation of $\beta$ - and $\varepsilon$-ring of $\alpha$-carotene, respectively, producing $\alpha$-branch xanthophylls (lutein), the most abundant carotenoids in light-harvesting complexes (LHCs) which is key structural and functional components of light harvesting $[6,8,26,33,37,39,59,72]$. During early stages $(0-4 \mathrm{~h})$ of HL (blue and white) treatments, lutein concentrations were lower than that of the control [84], which is listed in Figure S5 for details [see Additional file 9: Figure S5]. This result is consistent with the decreased transcriptional levels of Haecyp97a and Haecyp97c observed in $H$. pluvialis (Figure 6B and D). Although Haecyp97a and 
Haecyp 97 c transcriptional levels increased over time, lutein concentration level remained rather stable but was lower than the control. When considering that CYP97A is also involved in hydroxylation of $\beta$-carotene into zeaxanthin, the level of Haecyp $97 a$ increase is understandable [26]. Previous studies have demonstrated there was a cytochrome $\mathrm{P} 450$ involved in astaxanthin biosynthesis in $H$. pluvialis by the use of ellipticine [90]. Therefore, we speculate that CYP97A may be the cytochrome P450 involved in astaxanthin biosynthesis in $H$. pluvialis. According to our results, however, it is unclear why and how the levels of Haecyp $97 b$ and Haecyp97c increased. The function of CYP97B is currently unknown and they (CYP97B and CYP97C) may play some additional roles (aside from $\mathrm{CHY} \varepsilon$-ring) in algae under adverse conditions. An earlier study reported that cytochrome P450 reductase is also co-up-regulated with enzymes involved in DNA repair under light/dark cycles [91].

Our data (Figure 6) reveal that mRNA levels of different chy genes increase rapidly under HL exposure and varied with light wavelength. For instance, our results indicate that blue HL very significantly increased Haebch and Haecyp 97 c genes expression, and produced similar effects on Haecyp $97 a$ and Haecyp $97 b$ genes expression compare with white HL treatment. Young et al. [40] have demonstrated that different carotenoid metabolic gene isoforms typically had distinctly different expression patterns. Previous study has demonstrated that zeaxanthin and astaxanthin concentrations were more efficiently enhanced for $H$. pluvialis under blue HL treatment than white [84]. Therefore, we propose that Haematococcus cells are more sensitive to blue induction compare with white. Similar phenomena were reported red Light Emitting Diodes (LEDs) operated at a relatively low light intensity were found to be suitable for cell growth; and LEDs emitting short wavelength (380-470 $\mathrm{nm}$ ) can induce morphological changes in $H$. pluvialis and enhance astaxanthin accumulation [92-94]. It is well known that strong light (blue) enhances astaxanthin accumulation, because astaxanthin is produced by $H$. pluvialis cells to protect cells from the strong intensity of the light [93]. Although blue light contains more energy than red light, according to Planck's law [95], in fact only energy associated to the S1 transition level of chlorophyll (Chl) can be used for photosynthesis [96]. On this bottom line, the fact that the efficiency of energy transfer from carotenoid to $\mathrm{Chl}$ a is far lower (40\%) with respect to $\mathrm{Chl} \mathrm{b}$ to $\mathrm{Chl}$ a or $\mathrm{Chl}$ a to $\mathrm{Chl}$ a. Thus it can easily be concluded that less energy used for photosynthesis is available from blue light than from red or white light under the same light intensity. According to above conclusions, we speculate that astaxanthin accumulation for $H$. pluvialis depends on not only light intensity, but also light quality (i.e. blue light might be a more useful wavelength to enhance astaxanthin accumulation in $H$. pluvialis under HL intensity). Therefore, we believe that the response of $H$. pluvialis Flotow 1844 to high light stress is a complicated process involving bch, Haecyp $97 a$, Haecyp $97 b$, and Haecyp97c.

\section{Conclusions}

Our study provided a genome-wide comparative analysis of genes encoding CHYs in algae, with a focus on their distribution, structure, evolution, origins, and expression. As part of this study, we provided a summary of currently known distributions of chy genes in eukaryotic photosynthetic algae, and constructed hypotheses regarding xanthophylls biosynthetic pathways. Genes encoding $\mathrm{BCHs}$ of green algae and higher plants were determined to be non-cyanobacterial origin, whereas those from red algae were derived from cyanobacteria. Genes involved in $\beta$-xanthophylls biosynthetic pathway in Chromalveolates remains unknown. CYP97B genes are likely an innovation of eukaryotic algae, whereas genes of CYP97A and CYP97C initiated by gene duplication events before the split of green algae and higher plants. The biosynthesis of $\alpha$-xanthophylls is characteristic of green algae and higher plants. Although transcriptional levels of $b c h$, Haecyp 97a, Haecyp97b, and Haecyp97c were up-regulated by blue and white HL treatments, the amplitude and kinetics of mRNA accumulation varied among different chy genes. Compare with white light, blue light may play a more important role in controlling green algal carotenoid biosynthesis. The response of $H$. pluvialis Flotow 1844 to different HL stresses is a complicated course involving bch, Haecyp97a, Haecyp97b, and Haecyp97c. The observed increase in Haecyp $97 b$ transcriptional level implies that this gene may be another carotenoid biosyntheticrelated gene, perhaps a novel chy gene in Chromalveolates. Further investigations are needed to test this hypothesis.

\section{Methods}

\section{Identification of chy genes encoding CHY proteins}

The genomes of 18 eukaryotic photosynthetic algae included Chlamydomonas reinhardtii, Chlorella sp. NC64A, Chlorella vulgaris, Coccomyxa sp. C-169, Volvox carteri, Micromonas pusilla, Micromonas sp. RCC299, Ostreococcus sp. RCC809, Ostreococcus tauri, Ostreococcus lucimarinus, Phaeodactylum tricornutum, Thalassiosira pseudonana, Fragilariopsis cylindrus, Aureococcus anophagefferens, Emiliania huxleyi, Guillardia theta and Bigelowiella natans CCMP2755 were obtained from the website of the DOE Joint Genome Institute (Walnut Creek, CA, USA; http://genome.jgi.doe. gov/). The genome of the red alga Cyanidioschyzon merolae was obtained from the C. merolae Genome Project (http://merolae.biol.s.u-tokyo.ac.jp). The protein coding 
sequences of each genome was fed into the program makeblastdb to create an organism-species database [97].

Two methods were applied to identify the putative $\mathrm{CHY}$ homologs genes. Firstly, we followed JGI's or the $C$. merolae Genome Project's annotation to determine the number of chy present in the algal genomes. Then, eight previously characterized CHYs from Haematococcus pluvialis [GenBank: BCH, ABB70496.1], Chlamydomonas reinhardtii [GenBank: BCH, AAX54907.1], Synechocystis sp. PCC 6803 [GenBank: CrtR, BAA17468.1] and Arabidopsis thaliana [GenBank: BCH1, sp|Q9SZZ8.1; BCH2, sp| Q9LTG0.1; CYP97A3, NP_564384.1; CYP97B3, NP_193247.2, and CYP97C1, NP_190881.2] were used to construct a query protein set. BLASTp [97,98] and HMMER [99] programs were then conducted locally to identify all chy genes in all 18 algal genomes using a threshold evalue of 1e-10. Finally, we manually checked the extracted proteins by SMART and Pfam analyses to avoid false positive hits that commonly arise during large-scale automated analyses. Putative chy genes found by this method were added to the query set for another round of BLASTp searches. This procedure was iterated until no newly retrieved sequences that belonged to chy homologs. Moreover, in order to check for false negatives, two HMM models [Pfam: PF04116] and [Pfam: PF00067] derived from known bch and cyp 97 genes were applied to search for genes encoding chy on all proteins encoded in the 18 algal genomes $[100,101]$. All translated protein sequences of CHYsencoding genes used in this paper were listed in more detail [see Additional file 2]. CHY proteins from higher plants and cyanobacteria were also downloaded from National Center for Biotechnology Information GenBank database and Cyanobase (http://bacteria.kazusa.or.jp/ cyanobase/).

\section{Multiple sequence alignment and phylogenetic analysis} Proteins identified by the BLAST and HMM searches were aligned using ClustalW $[102,103]$ with a gap opening penalty of 10 , a gap extension penalty of 0.2 , and Gonnet as the weight matrix. The SMART [104] and Pfam 26.0 [105] databases were applied to delete false positives. Phylogenetic trees were constructed using Maximum likelihood (ML) method that implemented in PhyML [106]. ML trees were built in particular models of amino acid substitutions chosen according to PROTTEST AIC results [107]. The Le and Gascuel evolutionary model [108] was selected to analyze the protein phylogenies by assuming an estimated proportion of invariant sites and a gamma correction (four categories). Bootstrap values (BS) were inferred from 400 replicates. Graphical representation and edition of the phylogenetic tree were performed with TreeDyn (v198.3) [109].

\section{Algal strains and culture conditions}

H. pluvialis strain Flotow 1844 was obtained from Culture Collection of Algae and Protozoa, Dunstaffnage Marine Laboratory, and maintained at the Biological Resources Laboratory, Yantai Institute of Costal Zone Research, Chinese Academy of Science. Algae were incubated in 250-ml erlenmeyer flasks, each containing 100-ml BBM, and placed in an illuminating incubator (Ningbo Jiangnan Instrument Factory, GXZ-380, Ningbo, China) under a light intensity of $25 \mu \mathrm{mol}$ photons $\mathrm{m}^{-2} \mathrm{~s}^{-1}$ in $14 \mathrm{~h}: 10 \mathrm{~h} \mathrm{di}$ urnal scheme at temperature of $22 \pm 1^{\circ} \mathrm{C}$ without aeration.

For high light stress conditions, exponentially growing cultures (cell density approximately $5 \times 10^{7}$ cells $\mathrm{ml}^{-1}$ ) were harvested, and then transferred to 500-ml erlenmeyer flasks (named, 1-9), each containing 250-ml BBM (fresh medium) under continuous exposure of white light $(390-770 \mathrm{~nm})$ or blue light $(420-500 \mathrm{~nm})$ in light intensity of $1,000 \mu \mathrm{mol}$ photons $\mathrm{m}^{-2} \mathrm{~s}^{-1}$ without day/ night cycle. Algal cells collected $(20-\mathrm{mL}$ sample at some selected time) were rinsed with $\mathrm{PBS}$, stored at $-80^{\circ} \mathrm{C}$ if not immediately used. All experimental chemicals and reagents were of analytical grade.

\section{Cloning and characterization of the CYP97 genes}

H. pluvialis strain flotow 1844 in the exponential growth phase were harvested. Total RNA was extracted from fresh cells of $H$. pluvialis using Trizol reagent (TaKaRa D9108B, Dalian, China) according to the user manual. RNA solutions were stored at $-80^{\circ} \mathrm{C}$, if not immediately used. First-strand cDNAs were synthesized from $2 \mu \mathrm{g}$ total RNA with PrimeScript ${ }^{\circledR}$ RT Enzyme Mix I (TaKaRa DRR047A, Dalian, China) according to the manufacturer's instructions.

The CODEHOP (Consensus-degenerate hybrid oligonucleotide primers) strategy (http://blocks.fhcrc.org/ blocks/codehop.html) was used to generate degenerate primers $[110,111]$ for the cloning of core partial sequences of HaeCYP97A/B/C basing on the highly conserved regions of predicted putative genes encoding CYP97 homologs from some green algae. The nucleotide sequences of the 3'- and 5'-ends of HaeCYP97A/B/C were amplified by the RACE method [112,113]. Genespecific primers were designed from the amplified core cDNA sequence of HaeCYP97A/B/C and their 3'- and 5'-ends were obtained using SMARTTM RACE CDNA Amplification Kit (Clontech) according to the manual. All primers used in this study were listed in more [see Additional file 5: Table S3]. The PCR products were resolved by electrophoresis on $1 \%$ agarose gel. The fragment of interest was excised and purified using agarose gel DNA fragment recovery kit (TaKaRa D823A, Dalian, China). Finally, the fragment was cloned into PMD-18T vector (TaKaRa D101A, Dalian, China) and sequenced (Invitrogen, Beijing, China). 
The full-length cDNA sequence of HaeCYP97A/B/C was spliced according to the RACE-PCR results by the SeqMan software of DNAStar 7.1 (DNASTAR Inc., USA). The theoretical molecular weight $(\mathrm{Mw})$ and isoelectric point (pI) of HaeCYP97A/B/C protein were computed by ExPASy Compute pI/Mw tool [114]. Transmembrane regions were predicted by "DAS"-Transmembrane Prediction server [115]. Prediction of sub-cellular localization of the deduced amino acids was conducted using ChloroP and TargetP Servers $[82,83]$.

\section{Gene expression profiling: Real-time RT-PCR}

Total RNA from different samples was extracted using Trizol reagent (TaKaRa D9108B, Dalian, China) according to the user manual. Total RNA was treated with RNase-free DNase I (Fermentas, Glen Burnie, MD) to remove any residual genomic DNA that might be carried through the extraction process. Nuclear acids were quantified by NanoDrop 2000c (Thermo Scientific, USA). The first-strand cDNA synthesis for quantitative Real-time RT-PCR (qRT-PCR) was obtained from $1 \mu \mathrm{g}$ total RNA with PrimeScript ${ }^{\circledR}$ RT Enzyme Mix I (TaKaRa DRR047A, Dalian, China) according to the manufacturer's instructions.

Gene-specific primers were designed with Primer5 and are listed in more [see Additional file 5: Table S3]. All primer pairs were initially tested by standard RT-PCR and the amplification of single products of the correct size was verified on $2 \%(\mathrm{w} / \mathrm{v})$ agarose gels (data not shown). The actin gene [116] has been proven experimentally in this study [see Additional file 10: Figure S6]. The qRT-PCR amplifications were carried out in triplicate in a total volume of $20 \mu \mathrm{L}$ according to the manufacturer's instructions of SYBR ${ }^{\circledR}$ Premix Ex Taq ${ }^{\mathrm{Tm}}$ (Tli RNaseH Plus) (TaKaRa DRR420A, Dalian, China). The qRT-PCR program was holding stage, $50^{\circ} \mathrm{C}$ for $20 \mathrm{~s}$ and $95^{\circ} \mathrm{C}$ for $10 \mathrm{~min}$, followed by 40 cycles of $95^{\circ} \mathrm{C}$ for $15 \mathrm{~s}$, $60^{\circ} \mathrm{C}$ for $1 \mathrm{~min}$, and melt curve stage, $95^{\circ} \mathrm{C}$ for $15 \mathrm{~s}, 60^{\circ} \mathrm{C}$ for $1 \mathrm{~min}, 95^{\circ} \mathrm{C}$ for $30 \mathrm{~s}$, and $60^{\circ} \mathrm{C}$ for $15 \mathrm{~s}$. Real-time RT-PCR analysis was performed on an ABI fast 7500 Sequence Detection System (Applied Biosystems) following the protocol previously described using actin gene as the internal control. The relative steady state mRNA transcript levels were normalized to the respective actin transcripts. The $2^{-\Delta \Delta C T}$ method [117] was used to analyze quantitative real-time PCR data based on the cycle threshold $\left(C_{T}\right)$ values. The $\Delta \Delta C_{T}$ is represented as the following formula: $\Delta \Delta \mathrm{C}_{T}=\left(\mathrm{C}_{T}\right.$, target gene (test group) - $\mathrm{C}_{T}$, actin gene (test group)) time $\mathrm{x}-\left(\mathrm{C}_{T}\right.$, target gene (control group) $-C_{T}$, actin gene (control group)) times $\mathrm{x}$, where $\mathrm{x}$ is the time of selected sample. Therefore, the relative expression level of chy genes has been normalized to control group (normal light regime).

\section{Statistical analyses}

All exposure experiments were repeated three times independently, and data were recorded as the mean with standard deviation (SD). For gene expression experiments, quantitative real-time PCR analysis was performed using software BioRAD iQ5. For each gene, the expressed as the mean \pm SD (\% control) was calculated using the (standard curve) approximation corrected for primer efficiency and normalized to housekeeping gene actin expression values.

\section{Nucleotide sequencing and accession numbers}

The cDNA nucleotide sequences of HaeCYP97A/B/C have been deposited and assigned the accession number AFR31786, AFR36909, and AFQ31612, respectively, in the $\mathrm{EMB} / \mathrm{GenBank} / \mathrm{DDBJ}$ database.

\section{Additional files}

Additional file 1: Table S1. The details about the completeness of genome sequences used in this study. The details about the completeness of genome sequences used in this study were summarized from (DOE Joint Genome Institute, project list: http://genome.jgi.doe.gov/ genome-projects/). The genomes of the red alga Cyanidioschyzon merolae was obtained from the C. merolae Genome Project (http://merolae.biol.s. u-tokyo.ac.jp).

Additional file 2: The deduced protein sequences of genes encoding CHYs from 18 algal genomes. The red indicated genes encoding $\mathrm{BCH}$-type $\mathrm{CHYs}$ in algal genomes. The blue indicated two genes encoding proteins which belong to no one subfamily of CYP97.

Additional file 3: Table S2. The sequences information of $\mathrm{BCH}$ from higher plants and CrtRs from cyanobacteria was downloaded from NCBI database or Cyanobase respectively.

Additional file 4: Figure S1. The multiple sequence alignment of all CYP97 from algae. The names of each sequence are as listed in Additional file 2. A partial protein sequence (position: 272-926) has been selected for domain structure analysis.

Additional file 5: Table S3. List of primer sequences used for PCR amplification to clone three full-length CDNA of CYP97A/B/C homologs respectively in green alga Haematococcus pluvialis strain Flotow 1844. Note: F, forward; $R$, reverse; Position, the location of motifs where the primers were designed from protein multiple sequence alignments.

Additional file 6: Figure S2. Nucleotide and the predicted amino acid sequence of HaeCYP97A.

Additional file 7: Figure S3. Nucleotide and the predicted amino acid sequence of HaeCYP97B.

Additional file 8: Figure S4. Nucleotide and the predicted amino acid sequence of HaeCYP97C.

Additional file 9: Figure S5. Changes in total chlorophylls and carotenoids concentrations of $\mathrm{H}$. pluvialis under different high light stresses (white and blue). Cells grown in autotrophic medium were harvested and transferred to fresh medium with different high light intensity. Cells were harvested at different periods of induction and changes in total chlorophylls (A), total chlorophylls (B), lutein (C), acarotene (D), $\beta$-carotene (E), and astaxanthin (F). Values are mean $\pm S D$ of three independent determinations.

Additional file 10: Figure S6. The transcriptional level of actin gene under high light stress of different wavelength (blue and white).

\section{Abbreviations}

CHY: Carotenoid hydroxylase; $\mathrm{BCH}$ : Non-heme/di-iron $\beta$-ring hydroxylase; P450s: Cytochrome P450 proteins; CYP97: Cytochrome P450 protein, 97 
family; CD: Conserved domain; BS: Bootstrap value; RACE: Rapid Amplification of cDNA Ends; BKT: $\beta$-Carotenoid ketolase; HL: High light.

\section{Competing interests}

The authors have declared that no competing interests exist.

\section{Authors' contributions}

HLC and XNY conceived of the study, participated in all the bioinformatics analysis, including the sequence alignment and phylogeny analysis and drafted the manuscript; YLC helped in bioinformatics analysis, data mining and management; $X Q L$ and $Y W$ conceived the idea of identification of three full-length of CYP97 genes and designed the study; ZPL and SQ guided the writing of the manuscript. All authors read and approved the final manuscript.

\section{Acknowledgements}

This work was supported by the National Natural Science Foundation of China (No. 41176144); Ocean Public Welfare Scientific Research Project, State Oceanic Administration of China (No. 201205027); the Natural Science Foundation of Shandong Province (No. ZR2012DQ015); Project of the Guangdong Provincial Comprehensive Strategic Cooperation with the Chinese Academy of Sciences (No. 2011A090100040); Science and Technology Planning Project of Yantai, China (No. 2010247); and Nature Science Funds for Distinguished Young Scholar of Shandong Province (JQ200914).

\section{Author details}

${ }^{1}$ Key Laboratory of Coastal Biology and Biological Resources Utilization, Yantai Institute of Coastal Zone Research, Chinese Academy of Sciences, Yantai 264003, People's Republic of China. ' University of the Chinese Academy of Sciences, Beijing 100049, People's Republic of China. ${ }^{3}$ College of Resources and Environmental Sciences, Key Laboratory of Marine Biology, Nanjing Agricultural University, Nanjing 210095, People's Republic of China. ${ }^{4}$ Shenzhen Key Laboratory for Marine Bio-resource and Eco-environment, College of Life Sciences, Shenzhen University, Shenzhen 518060, People's Republic of China.

Received: 16 January 2013 Accepted: 12 June 2013

Published: 8 July 2013

\section{References}

1. Lichtenthaler HK: The 1-Deoxy-D-Xylulose-5-Phosphate pathway of isoprenoid biosynthesis in plants. Annu Rev Plant Physiol Plant Mol Biol 1999, 50(1):47-65.

2. Hirschberg J, Cohen M, Harker M, Lotan T, Mann V, Pecker I: Molecular genetics of the carotenoid biosynthesis pathway in plants and algae. Pure Appl Chem 1997, 69(10):2151-2158.

3. Niyogi KK: Photoprotection revisited: genetic and molecular approaches. Annu Rev Plant Biol 1999, 50(1):333-359.

4. Davison PA, Hunter CN, Horton P: Overexpression of beta-carotene hydroxylase enhances stress tolerance in Arabidopsis. Nature 2002, 418(6894):203-206.

5. DellaPenna D, Pogson BJ: Vitamin synthesis in plants: tocopherols and carotenoids. Annu Rev Plant Biol 2006, 57:711-738.

6. Dall'Osto L, Fiore A, Cazzaniga S, Giuliano G, Bassi R: Different roles of $a$ and $\beta$-branch xanthophylls in photosystem assembly and photoprotection. J Biol Chem 2007, 282(48):35056-35068.

7. Lokstein H, Tian L, Polle JE, DellaPenna D: Xanthophyll biosynthetic mutants of Arabidopsis thaliana: altered nonphotochemical quenching of chlorophyll fluorescence is due to changes in Photosystem II antenna size and stability. BBA 2002, 1553(3):309-319.

8. Tian L, Magallanes-Lundback M, Musetti V, DellaPenna D: Functional analysis of $\beta$-and $\varepsilon$-ring carotenoid hydroxylases in Arabidopsis. Plant Cell 2003, 15(6):1320-1332.

9. Niyogi KK, Grossman AR, Björkman O: Arabidopsis mutants define a central role for the xanthophyll cycle in the regulation of photosynthetic energy conversion. Plant Cell 1998, 10(7):1121-1134.

10. Pogson BJ, Niyogi KK, Björkman O, DellaPenna D: Altered xanthophyll compositions adversely affect chlorophyll accumulation and nonphotochemical quenching in Arabidopsis mutants. P Natl Acad Sci USA 1998, 95(22):13324-13329.

11. Niyogi KK, Shih C, Soon Chow W, Pogson BJ, DellaPenna D, Björkman O: Photoprotection in a zeaxanthin-and lutein-deficient double mutant of Arabidopsis. Photosynth Res 2001, 67(1):139-145.

12. Grossman AR: Paths toward algal genomics. Plant Physiol 2005, 137(2):410-427.

13. Rodríguez-Ezpeleta N, Brinkmann H, Burey SC, Roure B, Burger G, Löffelhardt W, Bohnert HJ, Philippe H, Lang BF: Monophyly of primary photosynthetic eukaryotes: green plants, red algae, and glaucophytes. Curr Biol 2005, 15(14):1325-1330.

14. Chan CX, Yang EC, Banerjee T, Yoon HS, Martone PT, Estevez JM, Bhattacharya D: Red and green algal monophyly and extensive gene sharing found in a rich repertoire of red algal genes. Curr Biol 2011, 21(4):328-333.

15. Moreira D, Le Guyader $H$, Philippe $H$ : The origin of red algae and the evolution of chloroplasts. Nature 2000, 405(6782):69-72

16. McFadden Gl: Primary and secondary endosymbiosis and the origin of plastids. J Phycol 2002, 37(6):951-959.

17. Martin W, Kowallik K: Annotated English translation of Mereschkowsky's 1905 paper 'Über Natur und Ursprung der Chromatophoren imPflanzenreiche'. Eur J Phycol 1999, 34(3):287-295.

18. Yoon HS, Hackett JD, Ciniglia C, Pinto G, Bhattacharya D: A molecular timeline for the origin of photosynthetic eukaryotes. Mol Biol Evol 2004, 21(5):809-818.

19. Lewis LA, McCourt RM: Green algae and the origin of land plants. Am J Bot 2004, 91(10):1535-1556.

20. Becker B, Marin B: Streptophyte algae and the origin of embryophytes. Ann Bot 2009, 103(7):999-1004.

21. Leliaert F, Verbruggen $H$, Zechman FW: Into the deep: new discoveries at the base of the green plant phylogeny. Bioessays 2011, 33(9):683-692.

22. Keeling PJ: The endosymbiotic origin, diversification and fate of plastids. Philos T Roy Soc B 2010, 365(1541):729-748.

23. Parker MS, Mock T, Armbrust EV: Genomic insights into marine microalgae. Annu Rev Genet 2008, 42:619-645.

24. McFadden Gl, van Dooren GG: Evolution: red algal genome affirms a common origin of all plastids. Curr Biol 2004, 14(13):R514-R516.

25. Keeling PJ: Diversity and evolutionary history of plastids and their hosts. Am J Bot 2004, 91(10):1481-1493.

26. Kim J, Smith JJ, Tian L, DellaPenna D: The evolution and function of carotenoid hydroxylases in Arabidopsis. Plant Cell Physiol 2009, 50(3):463-479.

27. Thayer SS, Björkman O: Leaf xanthophyll content and composition in sun and shade determined by HPLC. Photosynth Res 1990, 23(3):331-343.

28. Adams WW, Demmig-Adams B, Lange OL: Carotenoid composition and metabolism in green and blue-green algal lichens in the field. Oecologia 1993, 94(4):576-584

29. Hu Q, Miyashita H, Iwasaki I, Kurano N, Miyachi S, Iwaki M, Itoh S: A photosystem I reaction center driven by chlorophyll $\mathrm{d}$ in oxygenic photosynthesis. P Natl Acad Sci USA 1998, 95(22):13319-13323.

30. Stickforth P, Steiger S, Hess WR, Sandmann G: A novel type of lycopene $\varepsilon$-cyclase in the marine cyanobacterium Prochlorococcus marinus MED4. Arch Microbiol 2003, 179(6):409-415.

31. Schubert N, García-Mendoza E, Pacheco-Ruiz l: Carotenoid composition of marine red algae. J Phycol 2006, 42(6):1208-1216.

32. Yoshii $Y$ : Diversity and evolution of photosynthetic antenna systems in green plants. Phycol Res 2006, 54(3):220-229.

33. Kim J, DellaPenna D: Defining the primary route for lutein synthesis in plants: the role of Arabidopsis carotenoid $\beta$-ring hydroxylase CYP97A3. P Natl Acad Sci USA 2006, 103(9):3474-3479.

34. Sun $Z$, Gantt $E$, Francis $X \mathrm{Jr}$ : Cloning and functional analysis of the $\beta$-carotene hydroxylase of Arabidopsis thaliana. J Biol Chem 1996, 271(40):24349-24352.

35. Quinlan RF, Jaradat TT, Wurtzel ET: Escherichia coli as a platform for functional expression of plant P450 carotene hydroxylases. Arch Biochem Biophys 2007, 458(2):146-157.

36. Tian L, DellaPenna D: Characterization of a second carotenoid $\beta$-hydroxylase gene from Arabidopsis and its relationship to the LUT1 locus. Plant Mol Biol 2001, 47(3):379-388.

37. Tian L, Musetti V, Kim J, Magallanes-Lundback M, DellaPenna D: The Arabidopsis LUT1 locus encodes a member of the cytochrome P450 
family that is required for carotenoid $\varepsilon$-ring hydroxylation activity. P Natl Acad Sci USA 2004, 101(1):402-407.

38. Nelson D, Werck-Reichhart D: A P450-centric view of plant evolution. Plant J 2011, 66(1):194-211.

39. Dall'Osto L, Lico C, Alric J, Giuliano G, Havaux M, Bassi R: Lutein is needed for efficient chlorophyll triplet quenching in the major LHCII antenna complex of higher plants and effective photoprotection in vivo under strong light. BMC Plant Biol 2006, 6(1):32.

40. Young PR, Lashbrooke JG, Alexandersson E, Jacobson D, Moser C, Velasco R, Vivier MA: The genes and enzymes of the carotenoid metabolic pathway in Vitis vinifera L. BMC Genomics 2012, 13(1):243.

41. Tan CP, Zhao FQ, Su ZL, Liang CW, Qin S: Expression of $\beta$-carotene hydroxylase gene (crtR-B) from the green alga Haematococcus pluvialis in chloroplasts of Chlamydomonas reinhardtii. J Appl Phycol 2007, 19(4):347-355.

42. Linden H: Carotenoid hydroxylase from Haematococcus pluvialis: CDNA sequence, regulation and functional complementation. BBA-Gene Struct Expr 1999, 1446(3):203-212.

43. Lohr M, Im CS, Grossman AR: Genome-based examination of chlorophyll and carotenoid biosynthesis in Chlamydomonas reinhardtii. Plant Physiol 2005, 138(1):490-515.

44. Takaichi S: Carotenoids in algae: distributions, biosyntheses and functions. Mar Drugs 2011, 9(6):1101-1118.

45. Inoue K: Carotenoid hydroxylation-P450 finally! Trends Plant Sci 2004, 9(11):515-517.

46. Zhang X, Ye N, Liang C, Mou S, Fan X, Xu J, Xu D, Zhuang Z: De novo sequencing and analysis of the Ulva linza transcriptome to discover putative mechanisms associated with its successful colonization of coastal ecosystems. BMC Genomics 2012, 13(1):565.

47. Li Y, Sommerfeld M, Chen F, Hu Q: Effect of photon flux densities on regulation of carotenogenesis and cell viability of Haematococcus pluvialis (Chlorophyceae). J Appl Phycol 2010, 22(3):253-263.

48. Zhekisheva M, Zarka A, Khozin-Goldberg I, Cohen Z, Boussiba S: Inhibition of astaxanthin synthesis under high irradiance dose not abolish triacylglycerol accumulation in the green alga Haematococcus pluvialis (Chlorophyceae). J Phycol 2005, 41(4):819-826.

49. Lohr M, Wilhelm C: Xanthophyll synthesis in diatoms: quantification of putative intermediates and comparison of pigment conversion kinetics with rate constants derived from a model. Planta 2001, 212(3):382-391.

50. Lohr M, Wilhelm C: Algae displaying the diadinoxanthin cycle also possess the violaxanthin cycle. P Natl Acad Sci USA 1999, 96(15):8784-8789.

51. Coesel S, Oborník M, Varela J, Falciatore A, Bowler C: Evolutionary origins and functions of the carotenoid biosynthetic pathway in marine diatoms. PLoS One 2008, 3(8):e2896.

52. Tang KH, Barry K, Chertkov O, Dalin E, Han CS, Hauser LJ, Honchak BM, Karbach LE, Land ML, Lapidus A: Complete genome sequence of the filamentous anoxygenic phototrophic bacterium Chloroflexus aurantiacus. BMC Genomics 2011, 12(1):334.

53. Perry KL, Simonitch TA, Harrison-Lavoie KJ, Liu ST: Cloning and regulation of Erwinia herbicola pigment genes. J Bacteriol 1986, 168(2):607-612.

54. Misawa N, Nakagawa M, Kobayashi K, Yamano S, Izawa Y, Nakamura K, Harashima K: Elucidation of the Erwinia uredovora carotenoid biosynthetic pathway by functional analysis of gene products expressed in Escherichia coli. J Bacteriol 1990, 172(12):6704-6712.

55. Masamoto K, Misawa N, Kaneko T, Kikuno R, Toh H: r $\beta$ Carotene hydroxylase gene from the Cyanobacterium Synechocystis sp. PCC6803. Plant Cell Physio/ 1998, 39(5):560-564.

56. Fraser PD, Miura Y, Misawa N: In vitro characterization of astaxanthin biosynthetic enzymes. J Biol Chem 1997, 272(10):6128-6135.

57. Cunningham FX, Gantt E: A portfolio of plasmids for identification and analysis of carotenoid pathway enzymes: Adonis aestivalis as a case study. Photosynth Res 2007, 92(2):245-259.

58. Cunningham FX, Lee H, Gantt E: Carotenoid biosynthesis in the primitive red alga Cyanidioschyzon merolae. Eukaryot Cell 2007, 6(3):533-545.

59. Tian L, DellaPenna D: Progress in understanding the origin and functions of carotenoid hydroxylases in plants. Arch Biochem Biophys 2004, 430(1):22-29.

60. Bouvier F, Keller Y, d'Harlinque A, Camara B: Xanthophyll biosynthesis: molecular and functional characterization of carotenoid hydroxylases from pepper fruits (Capsicum annuum L.). BBA-Lipids and Lipid. Metabolism 1998, 1391(3):320-328.
61. Castillo R, Fernández JA, Gómez-Gómez L: Implications of carotenoid biosynthetic genes in apocarotenoid formation during the stigma development of Crocus sativus and its closer relatives. Plant Physiol 2005, 139(2):674-689.

62. Galpaz N, Ronen G, Khalfa Z, Zamir D, Hirschberg J: A chromoplast-specific carotenoid biosynthesis pathway is revealed by cloning of the tomato white-flower locus. Plant Cell 2006, 18(8):1947-1960.

63. Armbrust EV, Berges JA, Bowler C, Green BR, Martinez D, Putnam NH, Zhou S, Allen AE, Apt KE, Bechner M: The genome of the diatom Thalassiosira pseudonana: ecology, evolution, and metabolism. Science 2004, 306(5693):79-86.

64. Barbier G, Oesterhelt C, Larson MD, Halgren RG, Wilkerson C, Garavito RM, Benning C, Weber APM: Comparative genomics of two closely related unicellular thermo-acidophilic red algae, Galdieria sulphuraria and Cyanidioschyzon merolae, reveals the molecular basis of the metabolic flexibility of Galdieria sulphuraria and significant differences in carbohydrate metabolism of both algae. Plant Physiol 2005, 137(2):460-474.

65. Bowler C, Allen AE, Badger JH, Grimwood J, Jabbari K, Kuo A, Maheswari U, Martens C, Maumus F, Otillar RP: The Phaeodactylum genome reveals the evolutionary history of diatom genomes. Nature 2008, 456(7219):239-244.

66. Moktali V, Park J, Fedorova-Abrams N, Park B, Choi J, Lee YH, Kang S: Systematic and searchable classification of cytochrome P450 proteins encoded by fungal and oomycete genomes. BMC Genomics 2012, 13(1):525.

67. Initiative TAG: Analysis of the genome sequence of the flowering plant Arabidopsis thaliana. Nature 2000, 408(6814):796-815.

68. Ortiz De Montellano P, Voss J: Substrate oxidation by cytochrome P450 enzymes. Cytochrome 2005, P450:183-245.

69. Graham SE, Peterson JA: How similar are P450s and what can their differences teach us? Arch Biochem Biophys 1999, 369(1):24-29.

70. Werck-Reichhart D, Feyereisen R: Cytochromes P450: a success story. Genome Biol 2000, 1(6):3001-3003

71. Fiore A, Dall'Osto L, Cazzaniga S, Diretto G, Giuliano G, Bassi R: A quadruple mutant of Arabidopsis reveals a $\beta$-carotene hydroxylation activity for LUT1/CYP97C1 and a regulatory role of xanthophylls on determination of the PSI/PSII ratio. BMC Plant Biol 2012, 12(1):50.

72. Lv MZ, Chao DY, Shan JX, Zhu MZ, Shi M, Gao JP, Lin HX: Rice carotenoid $\beta$-ring hydroxylase CYP97A4 is involved in lutein biosynthesis. Plant Cell Physiol 2012, 53(6):987-1002.

73. Schuler MA, Werck-Reichhart D: Functional genomics of P450s. Annu Rev Plant Biol 2003, 54(1):629-667.

74. Cui H, Wang Y, Qin S: Molecular evolution of lycopene cyclases involved in the formation of carotenoids in eukaryotic algae. Plant Mol Biol Rep 2011, 29(4):1013-1020.

75. Hess WR, Rocap G, Ting CS, Larimer F, Stilwagen S, Lamerdin J, Chisholm SW: The photosynthetic apparatus of Prochlorococcus: Insights through comparative genomics. Photosynth Res 2001, 70(1):53-71.

76. Krubasik P, Sandmann G: Molecular evolution of lycopene cyclases involved in the formation of carotenoids with ionone end groups. Biochem Soc T 2000, 28:806-810.

77. Partensky F, Hoepffner N, Li WKW, Ulloa O, Vaulot D: Photoacclimation of Prochlorococcus sp. (Prochlorophyta) strains isolated from the North Atlantic and the Mediterranean Sea. Plant Physiol 1993, 101(1):285-296.

78. Benveniste I, Tijet N, Adas F, Philipps G, Salaün JP, Durst F: CYP86A1 from Arabidopsis thaliana encodes a cytochrome P450-dependent fatty acid omega-hydroxylase. Biochem Bioph Res Co 1998, 243(3):688-693.

79. Kern R, Bauwe H, Hagemann M: Evolution of enzymes involved in the photorespiratory 2-phosphoglycolate cycle from cyanobacteria via algae toward plants. Photosynth Res 2011, 109(1):103-114.

80. Lynch M, Conery JS: The evolutionary fate and consequences of duplicate genes. Science 2000, 290(5494):1151-1155.

81. Moore RC, Purugganan MD: The evolutionary dynamics of plant duplicate genes. Curr Opin Plant Biol 2005, 8(2):122-128.

82. Zhang J: Evolution by gene duplication: an update. Trends Ecol Evol 2003 18(6):292-298

83. Tran LT, Taylor JS, Constabel CP: The polyphenol oxidase gene family in land plants: Lineage-specific duplication and expansion. BMC Genomics 2012, 13(1):395.

84. Cui H, Yu X, Wang Y, Cui Y, Li X, Liu Z, Qin S: Gene cloning and expression profile of a novel carotenoid hydroxylase (CYP97C) from the green alga Haematococcus pluvialis. J Appl Phycol: . in press. 
85. Emanuelsson $\mathrm{O}$, Brunak $\mathrm{S}$, von Heijne $\mathrm{G}$, Nielsen $\mathrm{H}$ : Locating proteins in the cell using TargetP, SignalP and related tools. Nat Protoc 2007, 2(4):953-971.

86. Emanuelsson O, Nielsen $\mathrm{H}$, Heijne GV: ChloroP, a neural network-based method for predicting chloroplast transit peptides and their cleavage sites. Protein Sci 1999, 8(5):978-984.

87. Jin E, Polle JEW, Lee HK, Hyun SM, Chang M: Xanthophylls in microalgae: From biosynthesis to biotechnological mass production and application. J Microbiol Biotechn 2003, 13(2):165-174.

88. Li Y, Huang J, Sandmann G, Chen F: High-light and sodium chloride stress differentially regulate the biosynthesis of astaxanthin in Chlorella zofingiensis (Chlorophyceae) 1. J Phycol 2009, 45(3):635-641.

89. Margalith PZ: Production of ketocarotenoids by microalgae. App/ Microbiol Biotechn 1999, 51(4):431-438.

90. Schoefs B, Rmiki NE, Rachidi J, Lemoine Y: Astaxanthin accumulation in Haematococcus requires a cytochrome $\mathrm{P} 450$ hydroxylase and an active synthesis of fatty acids. FEBS Lett 2001, 500(3):125-128.

91. Monnier A, Liverani S, Bouvet R, Jesson B, Smith JQ, Mosser J, Corellou F, Bouget FY: Orchestrated transcription of biological processes in the marine picoeukaryote Ostreococcus exposed to light/dark cycles. BMC Genomics 2010, 11(1):192.

92. Katsuda T, Lababpour A, Shimahara K, Katoh S: Astaxanthin production by Haematococcus pluvialis under illumination with LEDs. Enzyme Microb Tech 2004, 35(1):81-86.

93. Katsuda T, Shimahara K, Shiraishi H, Yamagami K, Ranjbar R, Katoh S: Effect of flashing light from blue light emitting diodes on cell growth and astaxanthin production of Haematococcus pluvialis. J Biosci Bioeng 2006, 102(5):442-446.

94. Lababpour A, Hada K, Shimahara K, Katsuda T, Katoh S: Effects of nutrient supply methods and illumination with blue light emitting diodes (LEDs) on astaxanthin production by Haematococcus pluvialis. J Biosci Bioeng 2004, 98(6):452-456

95. Bose SN: Planck's law and the light quantum hypothesis. J Astrophys Astr 1994, 15(3):3-11.

96. Walla PJ, Linden PA, Ohta K, Fleming GR: Excited-state kinetics of the carotenoid S1 state in LHC II and two-photon excitation spectra of lutein and $\beta$-carotene in solution: efficient car $\mathrm{S} 1 \rightarrow \mathrm{Chl}$ electronic energy transfer via hot S1 states? J Phys Chem A 2002, 106(10):1909-1916.

97. Altschul SF, Gish W, Miller W, Myers EW, Lipman DJ: Basic local alignment search tool. J Mol Biol 1990, 215(3):403-410.

98. Altschul SF, Madden TL, Schäffer AA, Zhang J, Zhang Z, Miller W, Lipman DJ: Gapped BLAST and PSI-BLAST: a new generation of protein database search programs. Nucleic Acids Res 1997, 25(17):3389-3402.

99. Finn RD, Clements J, Eddy SR: HMMER web server: interactive sequence similarity searching. Nucleic Acids Res 2011, 39(2):W29-W37.

100. Punta M, Coggill PC, Eberhardt RY, Mistry J, Tate J, Boursnell C, Pang N, Forslund K, Ceric G, Clements J: The Pfam protein families database. Nucleic Acids Res 2012, 40(D1):D290-D301.

101. Johnson LS, Eddy SR, Portugaly E: Hidden Markov model speed heuristic and iterative HMM search procedure. BMC Bioinformatics 2010, 11(1):431.

102. Larkin MA, Blackshields G, Brown NP, Chenna R, McGettigan PA, McWilliam H, Valentin F, Wallace IM, Wilm A, Lopez R: Clustal W and Clustal X version 2.0. Bioinformatics 2007, 23(21):2947-2948.

103. Thompson JD, Higgins DG, Gibson TJ: CLUSTAL W: improving the sensitivity of progressive multiple sequence alignment through sequence weighting, position-specific gap penalties and weight matrix choice. Nucleic Acids Res 1994, 22(22):4673-4680.

104. Schultz J, Milpetz F, Bork P, Ponting CP: SMART, a simple modular architecture research tool: identification of signaling domains. P Natl Acad Sci USA 1998, 95(11):5857-5864.

105. Bateman A, Coin L, Durbin R, Finn RD, Hollich V, Griffiths-Jones S, Khanna A, Marshall M, Moxon S, Sonnhammer ELL: The Pfam protein families database. Nucleic Acids Res 2004, 32(suppl 1):D138-D141.

106. Guindon S, Gascuel O: A simple, fast, and accurate algorithm to estimate large phylogenies by maximum likelihood. Syst Biol 2003, 52(5):696-704.

107. Abascal F, Zardoya R, Posada D: ProtTest: selection of best-fit models of protein evolution. Bioinformatics 2005, 21(9):2104-2105.

108. Le SQ, Gascuel O: An improved general amino acid replacement matrix. Mol Biol Evol 2008, 25(7):1307-1320
109. Chevenet F, Brun C, Bañuls AL, Jacq B, Christen R: TreeDyn: towards dynamic graphics and annotations for analyses of trees. BMC Bioinformatics 2006, 7(1):439.

110. Rose TM, Henikoff JG, Henikoff S: CODEHOP (COnsensus-DEgenerate hybrid oligonucleotide primer) PCR primer design. Nucleic Acids Res 2003, 31(13):3763-3766.

111. Rose TM, Schultz ER, Henikoff JG, Pietrokovski S, McCallum CM, Henikoff S: Consensus-degenerate hybrid oligonucleotide primers for amplification of distantly related sequences. Nucleic Acids Res 1998, 26(7):1628-1635.

112. Godet S, Hérault J, Pencreac'h G, Ergan F, Loiseau C: Isolation and analysis of a gene from the marine microalga Isochrysis galbana that encodes a lipase-like protein. J Appl Phycol 2012, 24(6):1547-1553.

113. Sambrook J, Russell DW: Molecular cloning: a laboratory manual. Cold Spring Harbor, New York: CSHL press; 2001

114. Bjellqvist B, Hughes GJ, Pasquali C, Paquet N, Ravier F, Sanchez JC, Frutiger S, Hochstrasser D: The focusing positions of polypeptides in immobilized $\mathrm{pH}$ gradients can be predicted from their amino acid sequences. Electrophoresis 1993, 14(1):1023-1031.

115. Cserzö M, Eisenhaber F, Eisenhaber B, Simon I: On filtering false positive transmembrane protein predictions. Protein Eng 2002, 15(9):745-752.

116. Zhang L, Fan Y, Shi F, Qin S, Liu B: Molecular cloning, characterization, and expression analysis of a cytosolic HSP90 gene from Haematococcus pluvialis. J Appl Phycol 2012, 24(6):1601-1612.

117. Livak KJ, Schmittgen TD: Analysis of relative gene expression data using Real-Time quantitative PCR and the $2^{-\Delta \Delta C T}$ method. Meth 2001, 25(4):402-408.

doi:10.1186/1471-2164-14-457

Cite this article as: Cui et al:: Evolutionary origins, molecular cloning and expression of carotenoid hydroxylases in eukaryotic photosynthetic algae. BMC Genomics 2013 14:457.

\section{Submit your next manuscript to BioMed Central and take full advantage of:}

- Convenient online submission

- Thorough peer review

- No space constraints or color figure charges

- Immediate publication on acceptance

- Inclusion in PubMed, CAS, Scopus and Google Scholar

- Research which is freely available for redistribution

Submit your manuscript at www.biomedcentral.com/submit
C Biomed Central 